\title{
Summary of Hydrogeologic, Water-Quality, and Biologic Data from Two Small Basins, Southeast Hillsborough County, Florida
}

By T.H. Thompson and P.A. Metz

\author{
U.S. GEOLOGICAL SURVEY
}

Open-File Report 89-395

Prepared in cooperation with the

FLORIDA INSTITUTE OF PHOSPHATE RESEARCH 
U.S. DEPARTMENT OF THE INTERIOR MANUEL LUJAN, JR., Secretary

U.S. Geological Survey

Dallas L. Peck, Director

For additional information write to:

District Chief

U.S. Geological Survey

Suite 3015

227 North Bronough Street

Tallahassee, Florida 32301
Copies of this report may be purchased from:

U.S. Geological Survey

Books and Open-File Reports

Federal Center, Building 810

Box 25425

Denver, Colorado 80225 


\section{CONTENTS}

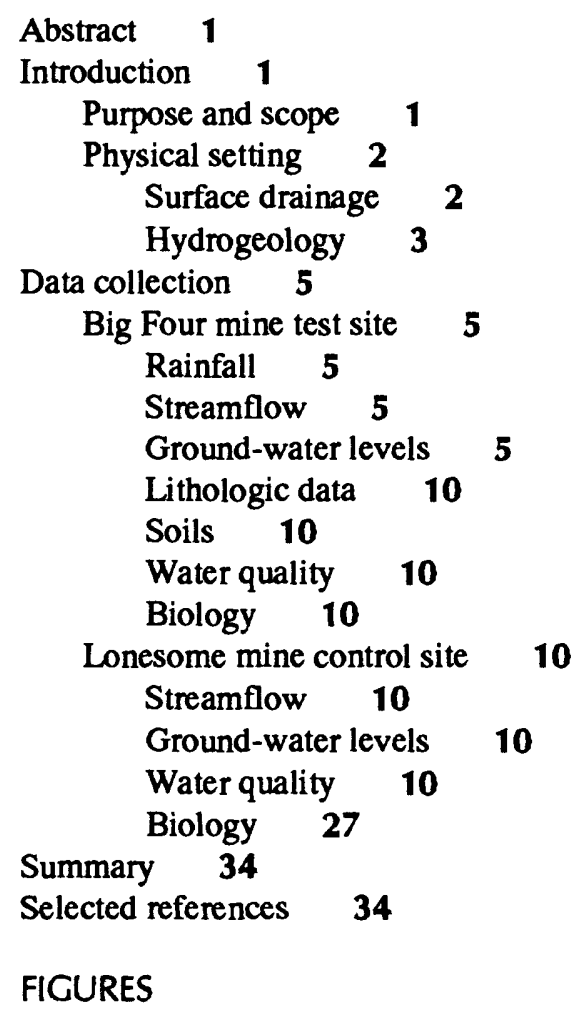

1-3. Maps showing:

1. Location of the study area in southeast Hillsborough County 2

2. Location of streamflow measuring sites in the Alafia and Little Manatee River basins

3. Premining data-collection network at the test site at the Big Four mine 6

4-6. Graphs showing:

4. Monthly rainfall at the Big Four mine test basin, June 1982 through July 1986

5. Water levels in well MM1, surficial aquifer, Big Four mine 15

6. Water levels in well MM3, intermediate aquifer, Big Four mine 15

7-8. Maps showing:

7. Data-collection network at the Big Four mine following regrading of former wetlands area at the test basin 16

8. Data-collection network at the control site at the Lonesome mine 27

9. Graph showing water levels at wells $\mathrm{CO}-3$ and $\mathrm{C}-4$ at the control basin, Lonesome mine

\section{TABLES}

1. Stratigraphic and hydrogeologic units of southeast Hillsborough County 4

2. Daily rainfall at the Big Four mine, June 1982 through September 19867

3. Daily discharge at transect B, Big Four mine, August 1982 through September 198611

4. Water-level observations at selected wells in the surficial aquifer system at Big Four mine $\quad 17$

5. Lithologic description of core 1 at transect A, Big Four mine 18 
6. Lithologic description of core 2 at transect $\mathrm{C}$, Big Four mine

7. Extractable chemical constituents and physical properties of soil samples, Big Four mine, August 3,1983

8. Water-quality data from selected wells and a stream at Big Four mine

20

9. Composition of flora at transect A, Big Four mine, 1983

23

10. Composition of flora at transects B and C, Big Four mine, 1983 through 1986

11. Benthic invertebrate dip-net survey at transects A, B, and C, Big Four mine 25

12. Daily discharge at the unnamed control basin tributary, Lonesome mine, July 1983 through September 1986 28

13. Water-level observations in the surficial aquifer system at the Lonesome mine near Fort Lonesome

14. Water-quality data from the unnamed tributary, Lonesome mine, September 23,1983

32

15. Composition of flora at transect AC, Lonesome mine, 1984 through 1986

\section{2}

16. Results of benthic invertebrate sampling using Hester-Dendy multiplated samplers at the unnamed tributary, Lonesome mine, April $1984 \quad 33$

\section{CONVERSION FACTORS}

The inch-pound units used in this report may be converted to metric (International System) units by the following factors:

\begin{tabular}{rrl}
\hline Multiply inch-pound units & By & To obtain metric units \\
\hline inch (in.) & 25.4 & millimeter $(\mathrm{mm})$ \\
inch per year (in $/ \mathrm{yr})$ & 25.4 & millimeter per year $(\mathrm{mm} / \mathrm{yr})$ \\
foot $(\mathrm{ft})$ & 0.3048 & meter $(\mathrm{m})$ \\
mile $(\mathrm{mi})$ & 1.609 & kilometer $(\mathrm{km})$ \\
square mile $\left(\mathrm{mi}^{2}\right)$ & 2.590 & square $\mathrm{kilometer}\left(\mathrm{km}^{2}\right)$ \\
$\mathrm{acre}$ & 0.4047 & hectare $($ ha) \\
cubic foot per second $\left(\mathrm{ft}^{3} / \mathrm{s}\right)$ & 0.02802 & cubic meter per second $\left(\mathrm{m}^{3} / \mathrm{s}\right)$ \\
foot squared per day $\left(\mathrm{ft}^{2} / \mathrm{d}\right)$ & 0.09290 & meter squared per day $\left(\mathrm{m}^{2} / \mathrm{d}\right)$ \\
gallon per minute $(\mathrm{gal} / \mathrm{min})$ & 0.06309 & liter per second $(\mathrm{L} / \mathrm{s})$
\end{tabular}

Sea level: In this report, "sea level" referes to the National Ceodetic Vertical Datum of 1929 (NCVD of 1929)-a geodetic datum derived from a general adjustment of the first-order level nets of both the United States and Canada, formerly called Sea Level Datum of 1929. 


\title{
Summary of Hydrogeologic, Water-Quality, and Biologic Data from Two Small Basins, Southeast Hillsborough County, Florida
}

\author{
By T.H. Thompson and P.A. Metz
}

\begin{abstract}
As part of a study to determine the environmental changes that might result from mining and reclamation, hydrologic, geologic, and biologic data were collected at two sites in southeast Hillsborough County between July 1983 and September 1986. Data were collected at the test basin at the Big Four mine before mining and following partial reclamation. Data also were collected at a nearby, unmined basin at the Lonesome mine. A summary of the data, which include rainfall, streamflow, ground-water levels, geologic data, physical and chemical data on soils, surface-water and ground-water quality, and blologic data, is presented.
\end{abstract}

\section{INTRODUCTION}

Florida produces about 80 percent of the Nation's supply of phosphate and 30 percent of the world's supply (Stowasser, 1986). Approximately 15 percent of the identified reserves lie beneath wetlands (J.W. Sweeney, U.S. Bureau of Mines, oral commun., 1983). In 1982, the U.S. Bureau of Mines and the U.S. Fish and Wildlife Service established memorandums of agreement with the Florida Institute of Phosphate Research to design and test reclamation strategies and to evaluate the success of reestablishment of disrupted wetlands. Critical factors in wetland ecosystem plant establishment and succession are depth of water, duration of surface inundation, and soil conditions within the range of tolerance for the desired vegetation types.

In 1982, the U.S. Geological Survey began a cooperative study with the Florida Institute of Phosphate Research to study the environmental aspects of mining and reclamation. The purpose of the study was to define the premining and postmining hydrology (water level and water quality), soil, and vegetative regimes of a proposed rockphosphate wetlands demonstration area, to relate postmining hydrologic conditions to the initial success or failure of revegetation efforts, and to provide baseline information for evaluating long-term wetlands revegetation.

Two basins in southeast Hillsborough County (fig. 1) were selected for monitoring. Premining data collection began at the test basin at the Big Four mine (fig. 2) in the summer of 1982. An unmined control basin at the Lonesome mine was instrumented and data collection began in 1983. Data from the control basin, approximately 5 miles southwest of the test basin (fig. 2), would indicate any major hydrologic or biologic changes not related to mining.

Mining commenced in October 1983 at the Big Four test site; however, there were numerous delays in the permitting process for reclamation. A permit was granted for restoration of the wetlands area only, and regrading of the wetlands part of the test basin started in May 1985. Planting of the wetlands vegetation was completed in January 1987. Regrading and vegetation planting of the remainder of the drainage basin ceased when mining operations were shut down in 1985.

\section{Purpose and Scope}

This report presents a summary of the hydrologic, geologic, water-quality, soils, and biologic data that were collected between July 1982 and September 1986 at the test and control sites in southeast Hillsborough County, Fla. The test basin at the Big Four mine had a premining drainage area of about 100 acres, of which 26 acres were classified as wetlands. All but 10 acres of wetlands were mined in 1985 . The control basin at the Lonesome mine has a drainage area of approximately 170 acres, including a wetlands area of about 10 acres. The control basin was not mined and generally remained unaffected by man's activities during the study period.

Because only 16 percent of the test basin had been reclaimed at the end of the study (September 1986), evaluation of the effects of mining and reclamation was postponed. This report is limited to presenting the data collected. 


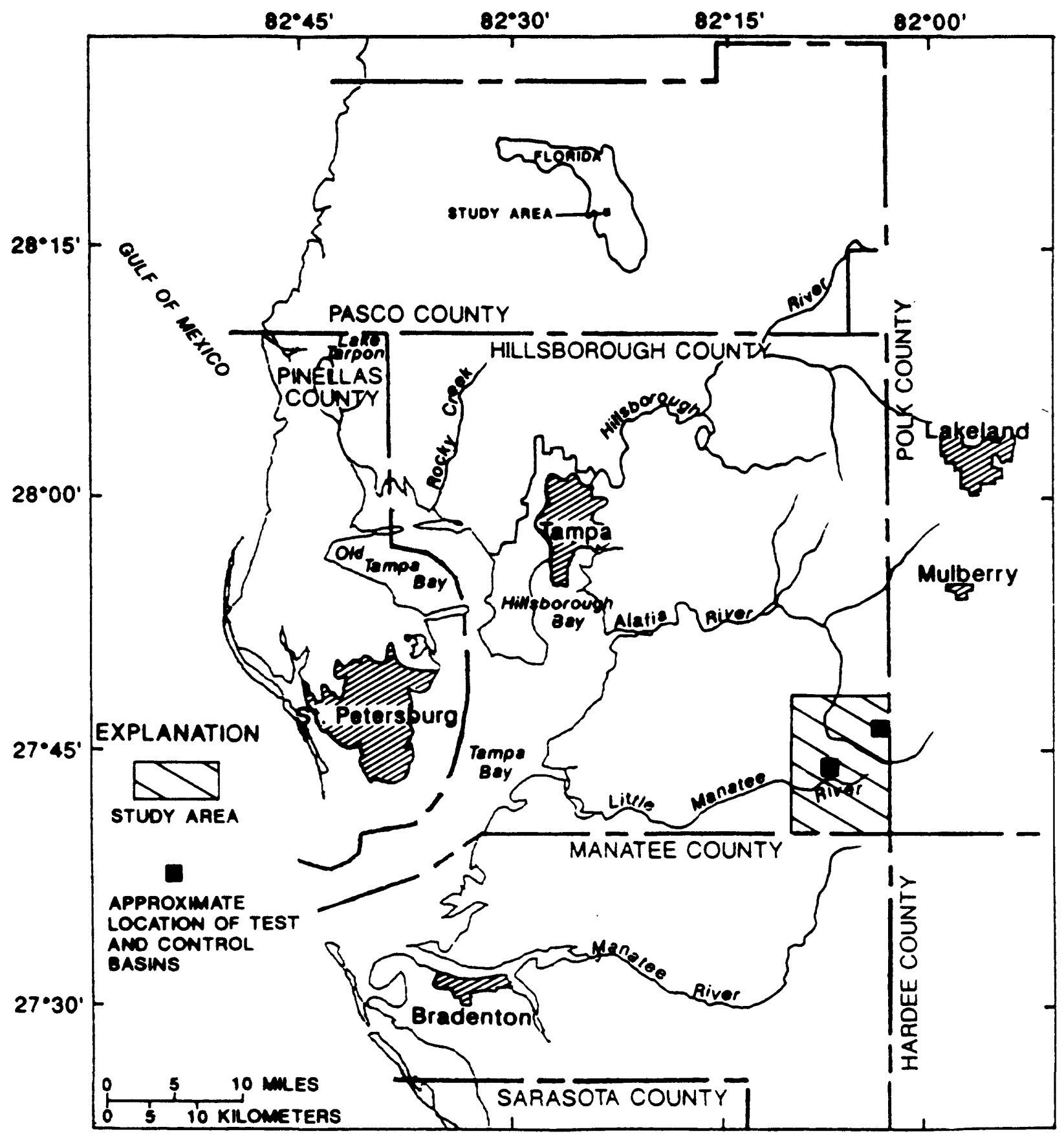

Figure 1. Location of the study area in southeast Hillsborough County.

\section{Physical Setting}

\section{Surface Drainage}

The study area is in the Polk Upland of the Central Highlands as defined by White $(1970$, p. 132). Land surface ranges in altitude from 100 to 130 feet. The area is underlain by siliclastic sediments that contribute to stream-channel development instead of the karst development that is typical in Pasco and Polk Counties to the north and east.

The test basin at the Big Four mine is tributary to Lake Branch, which is tributary to the South Prong of the Alafia River. Streamflow records for the South Prong Alafia River near Lithia have been collected at a gage 7.6 miles upstream 


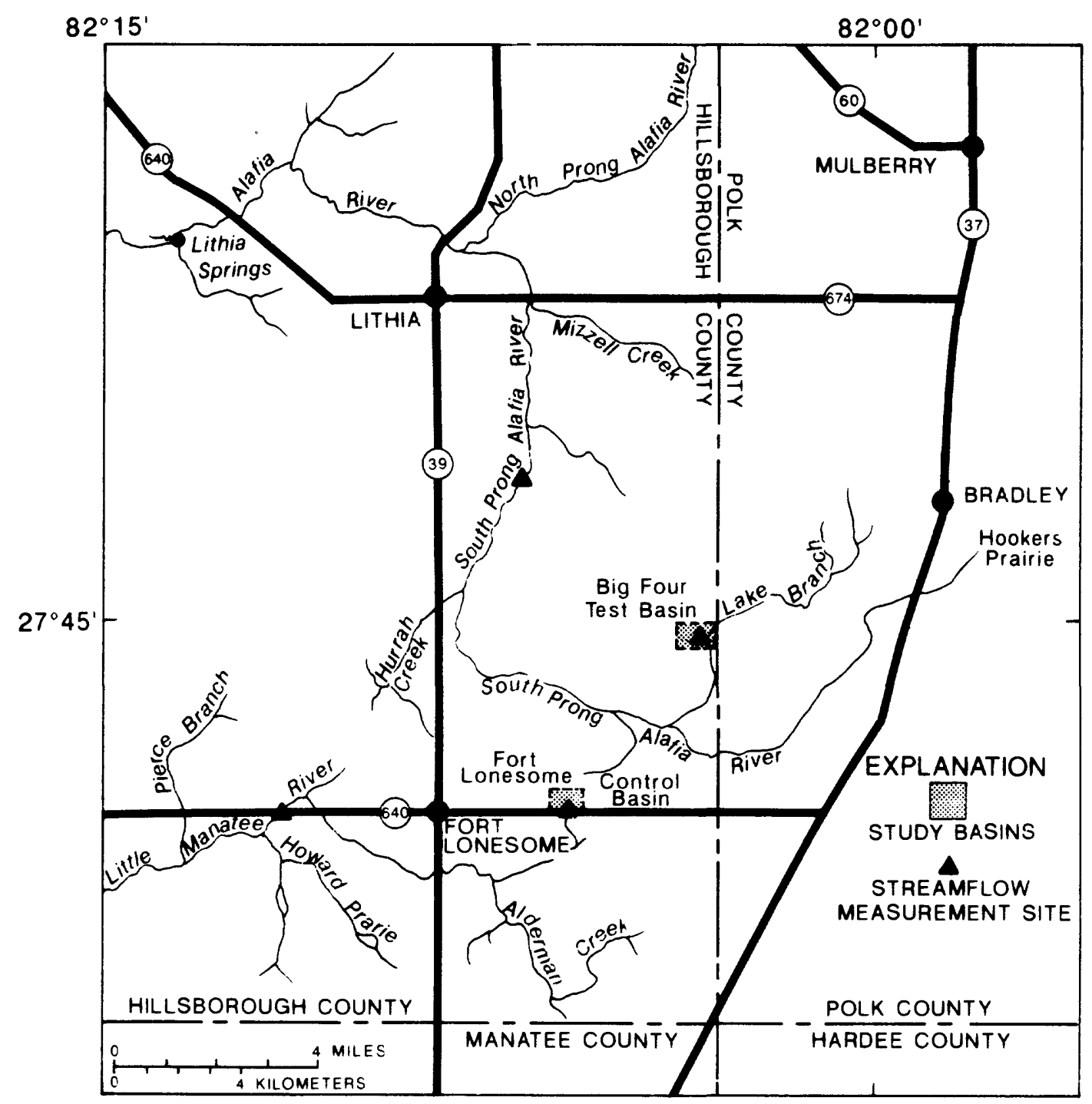

Figure 2. Location of streamflow measuring sites in the Alafia and Little Manatee River basins.

from the mouth (fig. 2) since December 1962. Average discharge for 24 years $(1963-86)$ is $103 \mathrm{ft}^{3} / \mathrm{s}$ from the $107-\mathrm{mi}^{2}$ drainage basin (13.08 in/yr). Maximum discharge of $2,600 \mathrm{ft}^{3} / \mathrm{s}$ for the period of record occurred on August 14, 1967. Minimum flow of $0.13 \mathrm{ft}^{3} / \mathrm{s}$ occurred on May 25,1981 .

The control basin at the Lonesome mine is tributary to the Little Manatee River. Streamflow records have been collected since September 1963 at a gage on the Little Manatee River near Fort Lonesome, 30 miles upstream from the mouth and 7 miles downstream from the control basin tributary (fig. 2). Average discharge for 23 years (1964-86) is $28.6 \mathrm{ft}^{3} / \mathrm{s}$ from the $31.4-\mathrm{mi}^{2}$ drainage basin $(12.37 \mathrm{in} / \mathrm{yr})$. Maximum discharge at the gage was $3,100 \mathrm{ft}^{3} / \mathrm{s}$ on September 22, 1979. There was no flow at times during most years of record.

\section{Hydrogeology}

Southeast Hillsborough County is underlain by a thick sequence of sedimentary rocks whose lithology and structure control the occurrence and movement of ground water. The principal stratigraphic and hydrogeologic units of the study area are shown in table 1. Stratigraphic units and their age, from youngest to oldest, are as follows: surficial deposits of Holocene and Pleistocene age; undifferentiated deposits of mostly Pliocene age; Hawthorn Formation and Tampa Limestone of Miocene age; Suwannee Limestone of Oligocene age; the Ocala Limestone, Avon Park Formation, and Oldsmar Formation of Eocene age; and the Cedar Keys Formation of Paleocene age. Principal hydrogeologic units 
Table 1. Stratigraphic and hydrogeologic units of southeast Hillsborough County

[Modified from Ryder, 1985, table 1]

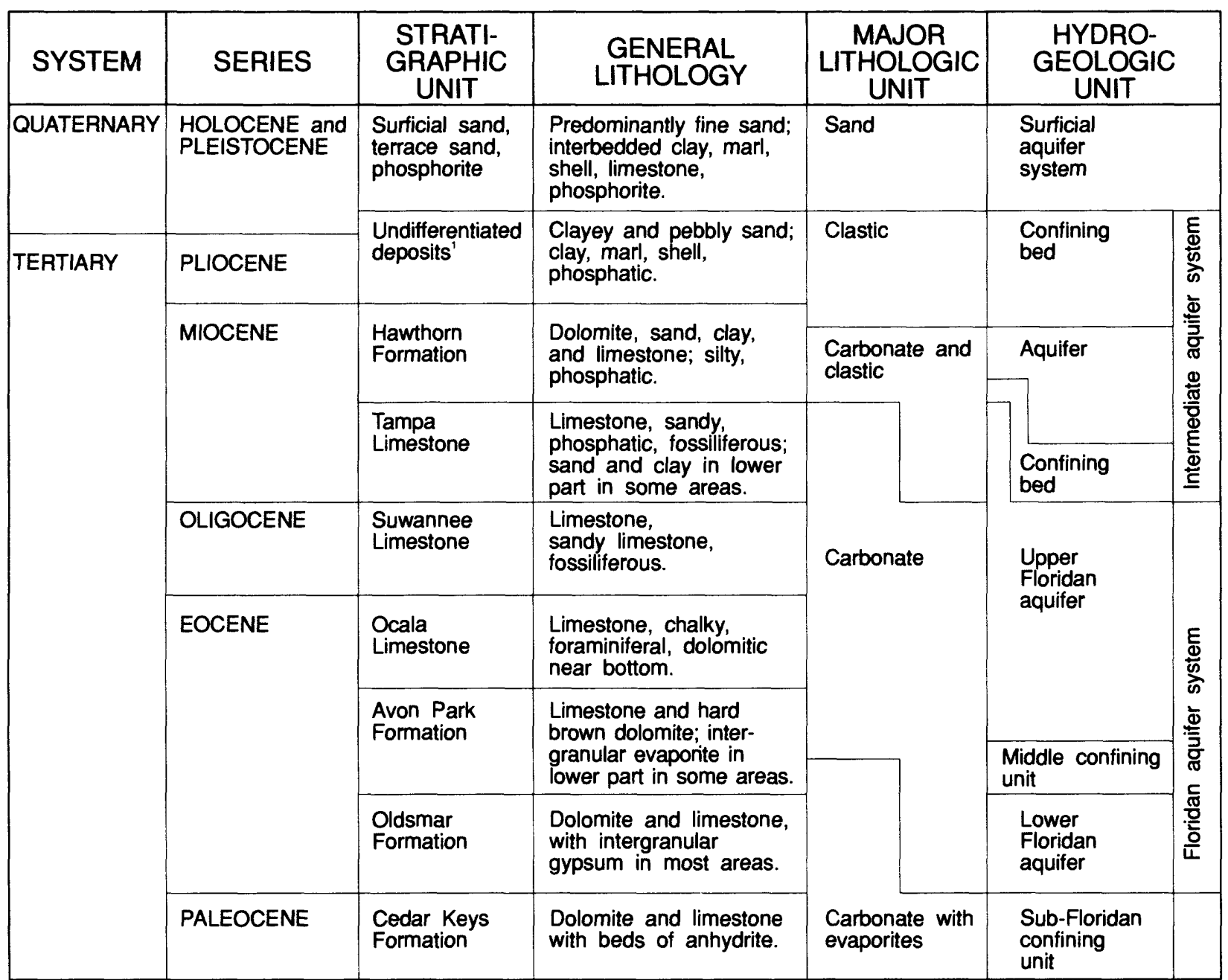

'Includes all or parts of Caloosahatchee Marl, Bone Valley Formation, and Tamiami Formation.

in the area consist of the surficial aquifer system, intermediate aquifer system, and the Floridan aquifer system (Southeastern Geological Society, 1986).

The surficial aquifer system consists of predominantly fine sand with interbedded clay, marl, shell, and limestone that are laterally discontinuous. The surficial aquifer system ranges in thickness from 20 to 40 feet in the study area. The surficial aquifer is used for lawn irrigation and stock watering. Small-diameter wells may yield up to $25 \mathrm{gal} / \mathrm{min}$.

The intermediate aquifer system in the study area consists of an upper confining bed, the lower Hawthornupper Tampa aquifer (Wolansky, 1983), and a lower confining bed. The confining units consist of clayey sand, silt, clay, and marl. The lower Hawthorn-upper Tampa aquifer consists of layers of limestone, dolomite, and sand, interbedded with clay and marl. The intermediate aquifer system is about 200 feet thick in the study area, according to Corral and Wolansky (1984), and is used for domestic supply and irrigation. Well yields range from 10 to $200 \mathrm{gal} / \mathrm{min}$.

The Floridan aquifer system has a total thickness of about 3,200 feet in the study area and includes three units: the Upper Floridan aquifer, the middle confining unit, and the Lower Floridan aquifer (Miller, 1986). The Upper Floridan aquifer is defined as a continuous sequence of carbonate 
rocks of Tertiary age that are hydraulically connected and generally have high permeability. In the study area, the Upper Floridan aquifer is about 1,200 feet thick and is the principal source of freshwater for irrigation and industrial (mining) use. Frequently, large-diameter supply wells are cased only to the first limestone (50-100 feet) and are open to pump water from both the intermediate aquifer system and the Upper Floridan aquifer. Wells may yield $2,000 \mathrm{gal} / \mathrm{min}$. The middle confining unit consists of carbonate rocks that contain intergranular evaporites of very low permeability. The unit is about 300 feet thick in southeast Hillsborough County. The Lower Floridan aquifer is made up of carbonate rocks of Eocene age and has low to very low permeability in the study area. Both the middle confining unit and the Lower Floridan aquifer contain saline water and are not used as a source of water for irrigation, industrial use, or public supply.

\section{DATA COLLECTION}

A reconnaissance of the Big Four mine test area to locate premining monitoring sites began in April 1982 and network installation began in May 1982. Two biological transects that were previously established by Biological Research Associates (1982) were reactivated. After the monitoring network at the Big Four mine was established, the search for a nearby control site was initiated. Several small tributaries to the Alafia River were examined but were eliminated from consideration because mining scheduled within 2 to 4 years would destroy or disturb the sites. The nearest suitable unmined control site was a small, unnamed tributary to the Little Manatee River at the Lonesome mine, about 5 miles southwest of the test site. Installation of a monitoring network at the control basin was started in September 1983.

\section{Big Four Mine Test Site}

The primary data-collection network at the Big Four mine test site consisted of a stream gage, a rain gage, two continuous-recording observation wells (MM3, deep; and MM1, shallow), seven shallow wells (MS1-MS4, MM2, MM4, and MM6) and one deep nonrecording observation well (MM5), two core-sample sites, six soil-sample sites, a stage-recording site in the wetlands, and three botantical transects (fig. 3). Benthic invertebrates were collected at the stream gage on transect $B$.

\section{Rainfall}

A continuous-recording rain gage was established at the top of a 30-foot berm at the upstream (western) edge of the drainage basin on May 27, 1982. Rainfall for the period June 1982 through July 1986 is shown in table 2 and figure 4. Annual rainfall for 1982 was 33.76 inches; for 1983, 58.19 inches; and for 1984, 37.76 inches. Normal annual rainfall at Bartow is 53.69 inches. Maximum daily precipitation was 3.85 inches on September 29, 1984.

\section{Streamflow}

The initial reconnaissance of the Big Four mine test site indicated that, under most flow conditions, the stream generally occupied more than one channel. A gaging site was located near transect $A$ where flow was generally confined to one channel, and a gage was established at that site on May 27, 1982 (fig. 3). A second gage was established just downstream of transect B on August 16, 1982, and the two gages were operated concurrently until sufficient data were available to develop a correlation between the flows at both sites. Streamflow measurements were discontinued at gage B when mining operations began in September 1983.

The site was reactivated when the berm around the wetlands was completed in January 1984. The gage stilling well was deepened following mining to monitor groundwater levels in the wetlands as well as stage during flow events. No flow occurred at the site until after the 16 acres of wetlands that were mined were regraded; the first flow following reclamation occurred on September 1, 1985, as a result of 3.65 inches of rainfall from Hurricane Elena. Streamflows for the periods August 1982 through September 1983 and January 1984 through September 1986 are shown in table 3.

\section{Ground-Water Levels}

A network of eight shallow wells (MS1-MS4, MM1, MM2, MM4, and MM5, depths less than 30 feet) and two deep wells (MM3 and MM6, depths greater than 50 feet) was established on June 4,1982, to monitor depth to water and changes in water levels in the surficial and intermediate aquifer systems (fig. 3). During dry periods, water levels ranged from 1.5 to 6.9 feet below land surface, and during the wet summer months, water levels ranged from at or near land surface to 4.1 feet below land surface. Water levels were monitored continuously in wells MM1 and MM3 in the surficial and intermediate aquifer systems, respectively, at transect $\mathrm{A}$. These data and periodic water-level measurements made at the surficial and intermediate aquifer system wells MM4 and MM6 at transect $C$ showed that their water levels were approximately the same and closely paralleled each other during changes in levels. These data indicate that the confining bed that separates the two aquifers may be discontinuous or is breached in the study area. Figures 5 and 6 show hydrographs of water levels in wells MM1 and MM3 open to the surficial and intermediate aquifer systems, respectively, for the period October 1, 1982, to September 30, 1983. 


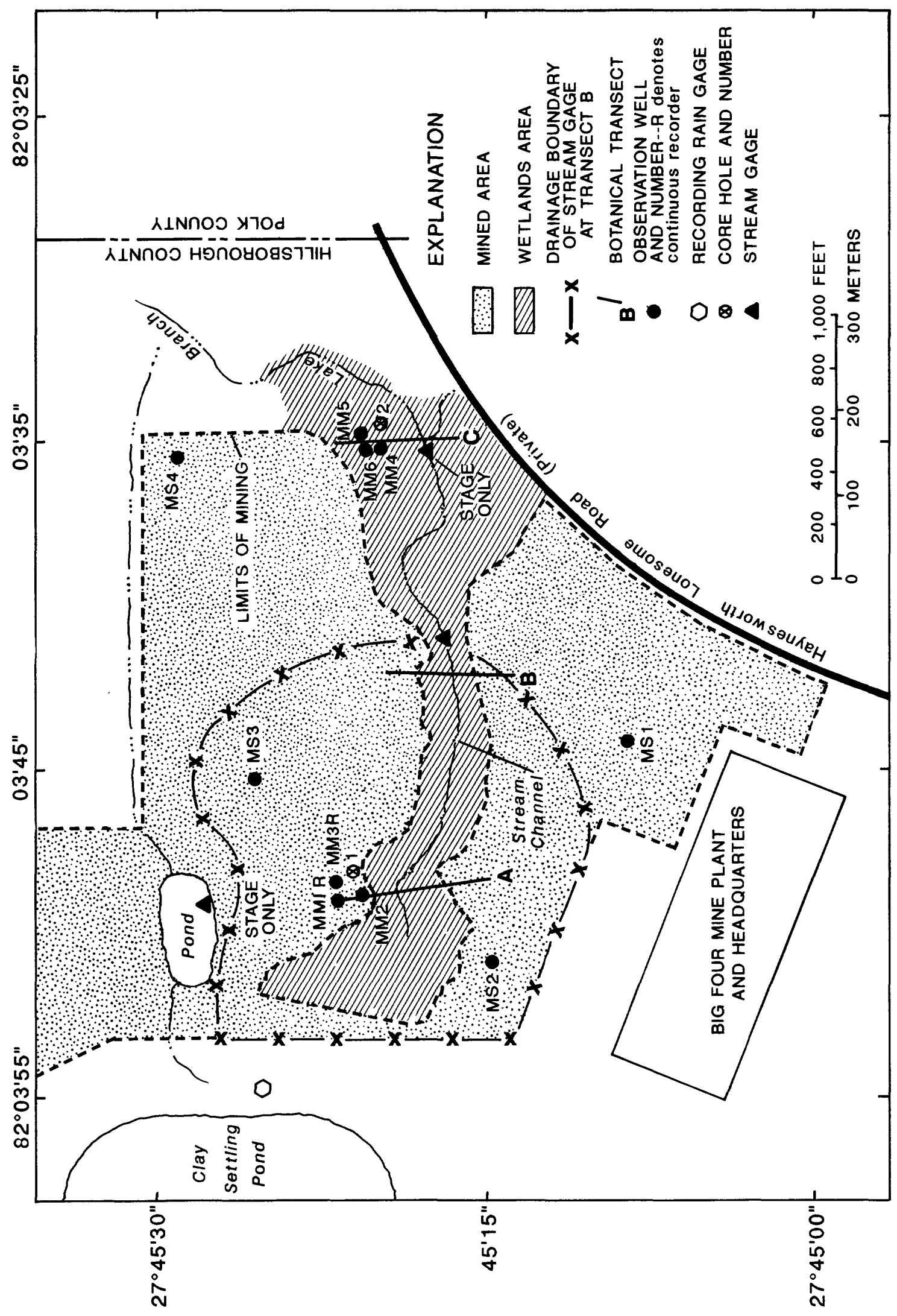

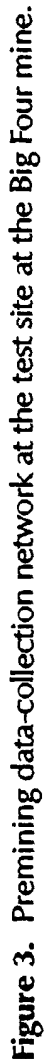




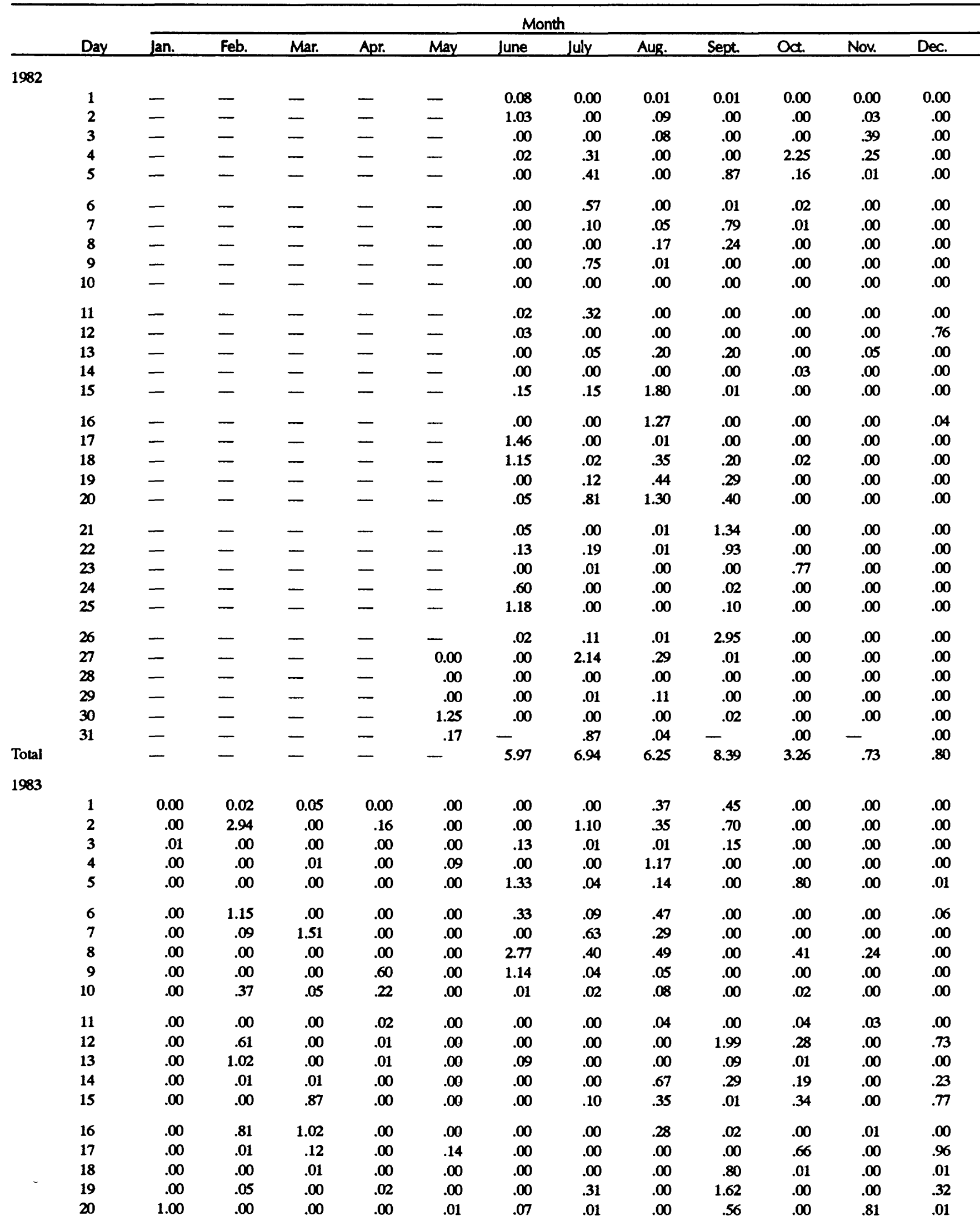


Table 2. Daily rainfall at the Big Four mine, June 1982 through September 1986-Continued

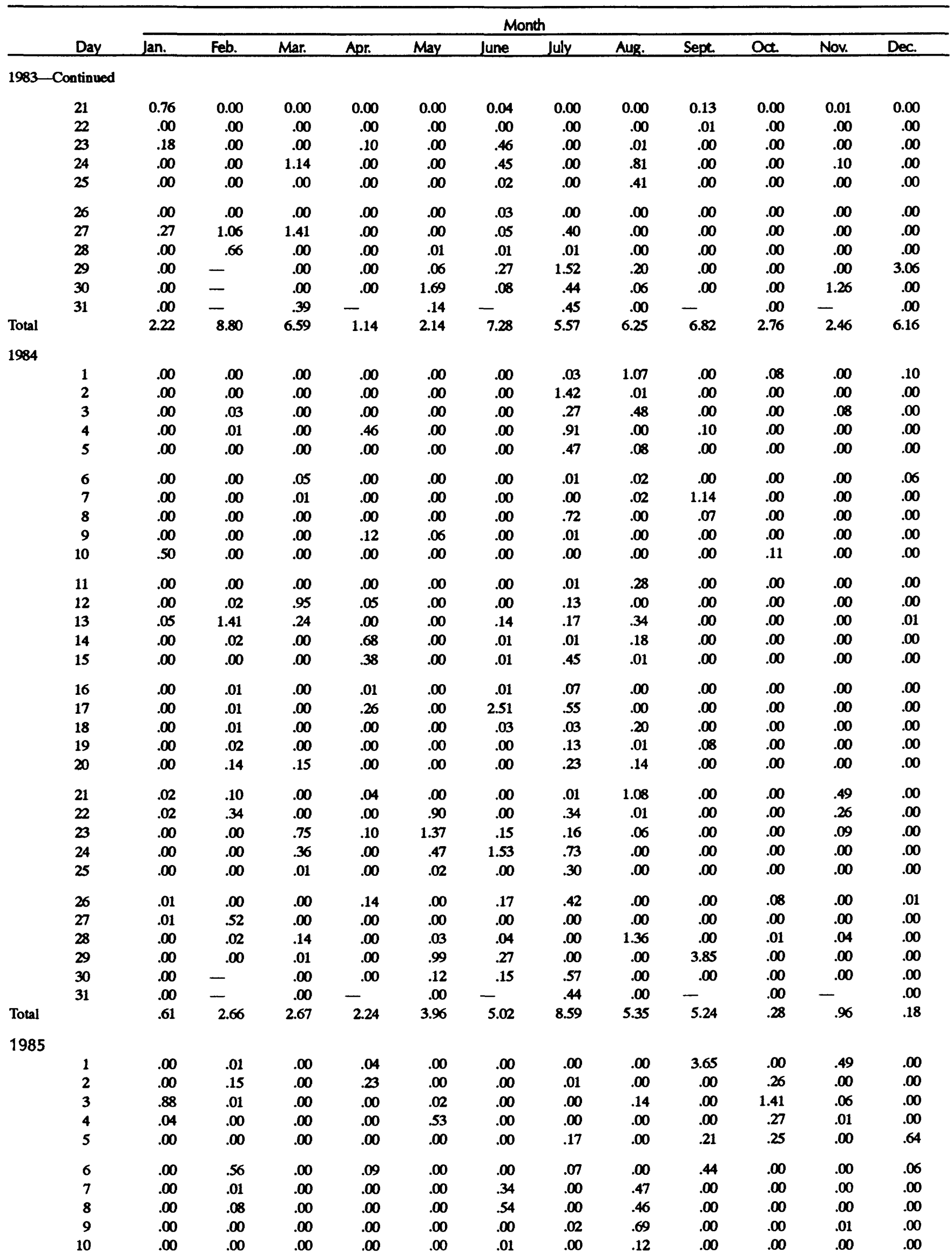


Table 2. Daily rainfall at the Big Four mine, June 1982 through September 1986—Continued

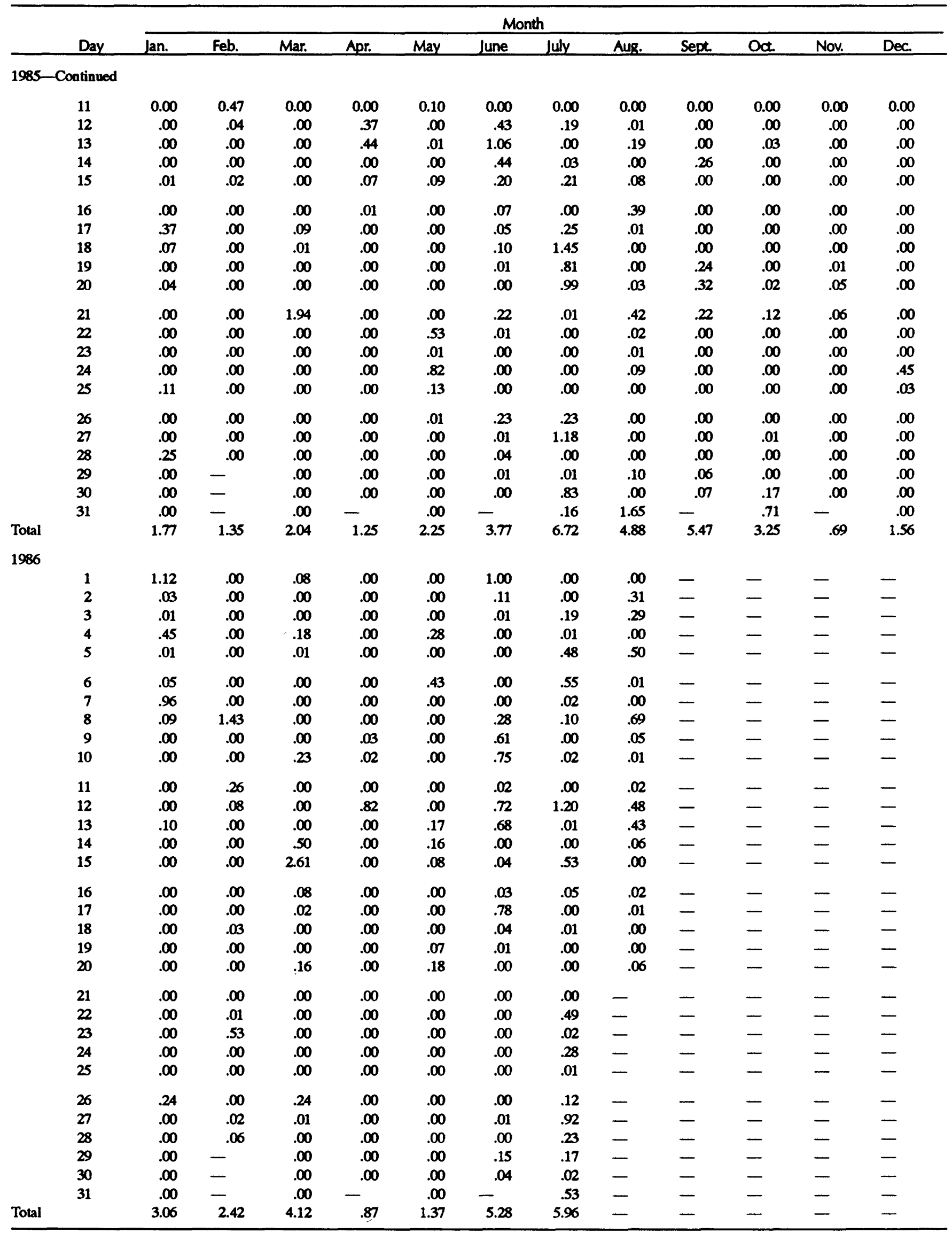




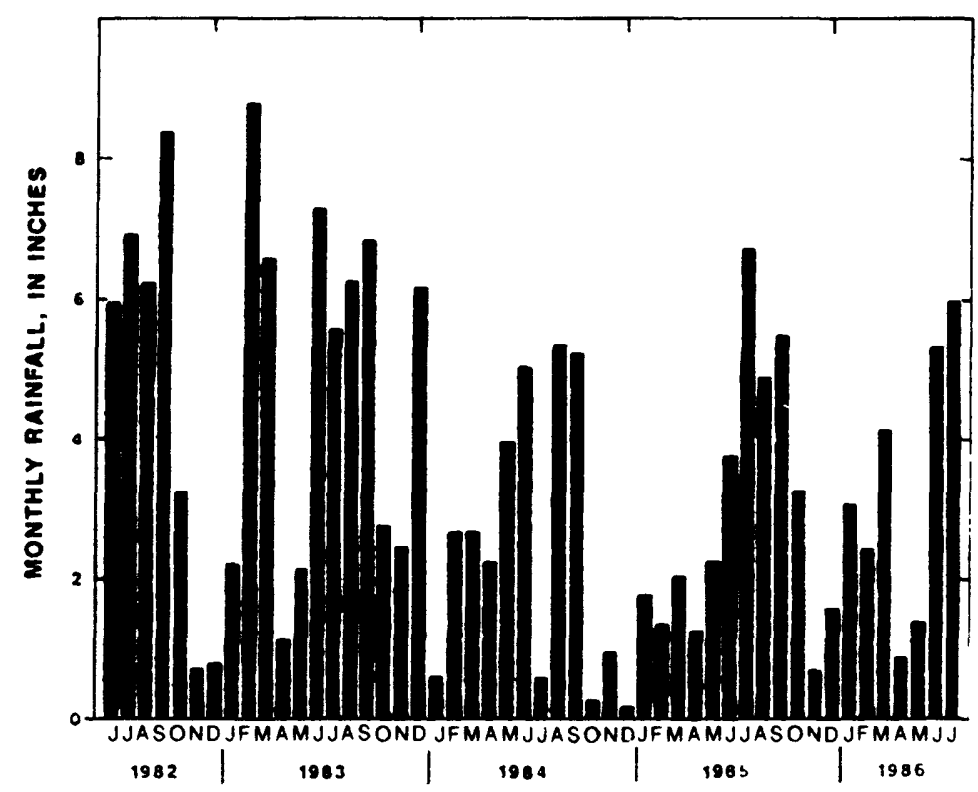

Figure 4. Monthly rainfall at the Big Four mine test basin, June 1982 through July 1986.

Table 3. Daily discharge at transect B, Big Four mine, August 1982 through September 1986 [All values are in cubic feet per second; - , no data]

\begin{tabular}{|c|c|c|c|c|c|c|c|c|c|c|c|c|}
\hline \multirow[b]{2}{*}{ Day } & \multicolumn{12}{|c|}{ Month } \\
\hline & Jan. & Feb. & Mar. & Apr. & May & June & July & Aug. & Sept. & Oct. & Nov. & Dec. \\
\hline \multicolumn{13}{|l|}{1982} \\
\hline 1 & - & - & - & - & - & - & - & - & 0.14 & 0.19 & 0.16 & 0.13 \\
\hline 2 & - & - & - & - & - & - & - & - & .14 & .17 & .16 & .11 \\
\hline 3 & - & - & - & - & - & - & - & - & .14 & .16 & .17 & .11 \\
\hline 4 & - & - & - & - & - & - & - & - & .11 & .66 & .23 & .11 \\
\hline 5 & - & - & - & - & - & - & - & - & .13 & 1.6 & .23 & .11 \\
\hline 6 & - & - & - & - & - & - & - & 0.14 & .29 & .45 & .21 & .10 \\
\hline 7 & - & - & - & - & - & - & - & .14 & .34 & .27 & .17 & .10 \\
\hline 8 & - & - & - & - & - & - & - & .14 & .55 & .19 & .16 & .10 \\
\hline 9 & - & - & - & - & - & - & - & .13 & .36 & .17 & .14 & .11 \\
\hline 10 & - & - & - & - & - & - & - & .13 & .27 & .16 & .13 & .10 \\
\hline 11 & - & - & - & - & _ & - & - & .11 & .23 & .13 & .11 & .10 \\
\hline 12 & - & - & - & - & - & - & - & .11 & .23 & .11 & .11 & .25 \\
\hline 13 & - & - & - & - & - & - & - & .10 & .23 & .11 & .13 & .14 \\
\hline 14 & - & - & - & - & - & - & - & .10 & .23 & .11 & .13 & .10 \\
\hline 15 & - & - & - & - & - & - & - & 1.4 & .23 & .11 & .13 & .10 \\
\hline 16 & - & - & - & - & - & - & - & 2.7 & .23 & .11 & .14 & .10 \\
\hline 17 & - & - & - & - & - & - & - & 1.2 & .23 & .10 & .14 & .10 \\
\hline 18 & - & - & - & - & - & - & - & .58 & .23 & .10 & .16 & .10 \\
\hline
\end{tabular}




\section{Month}

Day Jan. Feb. Mar. Apr. May June July Aug. Sept. Oct. Nov. Dec.

1982-Continued

\begin{tabular}{|c|c|c|c|c|c|c|c|c|c|c|c|c|}
\hline 19 & - & - & - & - & - & - & - & 0.96 & 0.23 & 0.10 & 0.14 & 0.10 \\
\hline 20 & - & - & - & - & - & - & - & 1.8 & .25 & .10 & .14 & .09 \\
\hline 21 & - & - & - & - & - & - & - & 1.9 & .87 & .10 & .14 & .08 \\
\hline 22 & - & - & - & - & - & - & - & .39 & 2.0 & .09 & .16 & .08 \\
\hline 23 & - & - & - & - & - & - & - & .29 & .58 & .14 & .17 & .07 \\
\hline 24 & - & - & - & - & - & - & - & .25 & .34 & .27 & .14 & .07 \\
\hline 25 & - & - & - & - & - & - & - & .18 & .27 & .19 & .14 & .07 \\
\hline 26 & - & - & - & - & - & - & - & .16 & 7.2 & .16 & .14 & .06 \\
\hline 27 & - & - & - & - & - & - & - & .16 & 1.4 & .14 & .14 & .06 \\
\hline 28 & - & - & - & - & - & & - & .17 & .27 & .13 & .13 & .06 \\
\hline 29 & - & - & - & - & - & - & - & .17 & .23 & .13 & .13 & .06 \\
\hline 30 & - & - & - & - & - & - & - & .16 & .21 & .13 & .13 & .06 \\
\hline 31 & - & - & - & - & - & - & - & .16 & - & .14 & - & .06 \\
\hline & - & - & - & - & - & - & - & - & 18.16 & 6.72 & 4.51 & 2.99 \\
\hline & - & - & - & - & - & - & - & - & .61 & .22 & .15 & .10 \\
\hline & - & - & - & - & - & - & - & - & 7.2 & 1.6 & .23 & .25 \\
\hline & - & - & - & - & - & - & - & - & .11 & .09 & .11 & .06 \\
\hline
\end{tabular}

1983

\begin{tabular}{|c|c|c|c|c|c|c|c|c|c|c|c|c|}
\hline 1 & .06 & .10 & .45 & .24 & .08 & .11 & .11 & .46 & .16 & - & - & - \\
\hline 2 & .06 & 2.2 & .23 & .21 & .08 & .10 & .25 & .42 & .29 & - & - & - \\
\hline 3 & .06 & .29 & .20 & .20 & .08 & .09 & .42 & .27 & .36 & - & - & - \\
\hline 4 & .06 & .17 & .20 & .17 & .07 & .09 & .23 & .57 & .21 & - & - & - \\
\hline 5 & .07 & .14 & .19 & .16 & .09 & .19 & .21 & .51 & .17 & - & - & - \\
\hline 6 & .08 & .32 & .19 & .14 & .08 & .20 & .19 & .47 & .14 & - & - & - \\
\hline 7 & .08 & .63 & .85 & .13 & .06 & .14 & .25 & .62 & .13 & - & - & - \\
\hline 8 & .08 & .22 & 1.0 & .14 & .04 & 1.8 & .48 & 1.1 & .13 & - & - & - \\
\hline 9 & .08 & .11 & .33 & .16 & .04 & 1.0 & .25 & .48 & .13 & - & - & - \\
\hline 10 & .07 & .12 & .28 & .75 & .04 & 1.3 & .19 & .27 & .11 & - & - & - \\
\hline 11 & .07 & .15 & .28 & .23 & .02 & .33 & .16 & .23 & .11 & - & - & - \\
\hline 12 & .07 & .14 & .26 & .18 & .02 & .21 & .14 & .21 & 1.4 & - & - & - \\
\hline 13 & .07 & 1.4 & .25 & .16 & .02 & .16 & .11 & .23 & .96 & - & - & - \\
\hline 14 & .06 & .31 & .19 & .16 & .02 & .17 & .11 & .27 & .29 & - & - & - \\
\hline 15 & .06 & .24 & .48 & .13 & .02 & .16 & .10 & .23 & .27 & - & - & - \\
\hline 16 & .06 & .83 & .46 & .11 & .02 & .14 & .10 & .19 & .22 & - & - & - \\
\hline 17 & .06 & .39 & 1.3 & .10 & .02 & .10 & .10 & .16 & .19 & - & - & - \\
\hline 18 & .06 & .26 & .23 & .09 & .01 & .10 & .10 & .16 & .55 & - & - & - \\
\hline 19 & .06 & .24 & .15 & .08 & .01 & .09 & .10 & .14 & 3.6 & - & - & - \\
\hline 20 & .31 & .21 & .12 & .08 & .01 & .09 & .10 & .14 & 1.3 & - & - & - \\
\hline 21 & 1.1 & .20 & .12 & .08 & .01 & .10 & .10 & .13 & .58 & - & - & - \\
\hline 22 & .42 & .19 & .12 & .08 & .01 & .09 & .08 & .13 & .34 & - & - & - \\
\hline 23 & .31 & .18 & .12 & .08 & .01 & .10 & .08 & .11 & .13 & - & - & - \\
\hline 24 & .27 & .18 & .72 & .07 & .01 & .13 & .08 & .21 & .13 & - & - & - \\
\hline 25 & .23 & .16 & .25 & .07 & .01 & .16 & .08 & .31 & .13 & - & - & - \\
\hline 26 & .10 & .16 & .20 & .07 & .01 & .13 & .08 & .29 & .11 & - & - & - \\
\hline 27 & .11 & .75 & 1.2 & .07 & .01 & .11 & .09 & .21 & .11 & - & - & - \\
\hline 28 & .13 & 1.0 & .66 & .07 & .01 & .10 & .10 & .16 & .11 & - & - & - \\
\hline 29 & .12 & - & .23 & .07 & .02 & .10 & .46 & .16 & .10 & - & - & - \\
\hline
\end{tabular}


Table 3. Daily discharge at transect B, Big Four mine, August 1982 through September 1986—Continued

\begin{tabular}{|c|c|c|c|c|c|c|c|c|c|c|c|c|c|}
\hline & \multirow[b]{2}{*}{ Day } & \multicolumn{12}{|c|}{ Month } \\
\hline & & Jan. & Feb. & Mar. & Apr. & May & June & July & Aug. & Sept. & Oct. & Nov. & Dec. \\
\hline \multicolumn{14}{|c|}{ 1983-Continued } \\
\hline & 30 & 0.12 & - & 0.20 & 0.08 & 0.12 & 0.14 & 0.45 & 0.17 & 0.10 & - & - & - \\
\hline & 31 & .10 & - & .26 & 一 & .17 & - & .51 & .16 & - & - & - & - \\
\hline Total & & 4.59 & 11.29 & 11.72 & 4.36 & 1.22 & 7.73 & 5.81 & 9.17 & 12.56 & - & - & - \\
\hline Mean & & .15 & .40 & .38 & .15 & .04 & .26 & .19 & .30 & .42 & - & - & - \\
\hline Maximum & & 1.1 & 2.2 & 1.3 & .75 & .17 & 1.8 & .51 & 1.1 & 3.6 & - & - & - \\
\hline Minimum & & .06 & .10 & .12 & .07 & .01 & .09 & .08 & .11 & .10 & - & - & - \\
\hline \multicolumn{14}{|l|}{1984} \\
\hline & 1 & - & .00 & .00 & - & .00 & .00 & .00 & .00 & .00 & 0.00 & 0.00 & 0.00 \\
\hline & 2 & - & .00 & .00 & - & .00 & .00 & .00 & .00 & .00 & .00 & .00 & .00 \\
\hline & 3 & - & .00 & .00 & - & .00 & .00 & .00 & .00 & .00 & .00 & .00 & .00 \\
\hline & 4 & - & .00 & .00 & - & .00 & .00 & .00 & .00 & .00 & .00 & .00 & .00 \\
\hline & 5 & - & .00 & .00 & - & .00 & .00 & .00 & .00 & .00 & .00 & .00 & .00 \\
\hline & 6 & - & .00 & .00 & - & .00 & .00 & .00 & .00 & .00 & .00 & .00 & .00 \\
\hline & 7 & - & .00 & .00 & - & .00 & .00 & .00 & .00 & .00 & .00 & .00 & .00 \\
\hline & 8 & - & .00 & .00 & - & .00 & .00 & .00 & .00 & .00 & .00 & .00 & .00 \\
\hline & 9 & - & .00 & .00 & - & .00 & .00 & .00 & .00 & .00 & .00 & .00 & .00 \\
\hline & 10 & - & .00 & .00 & - & .00 & .00 & .00 & .00 & .00 & .00 & .00 & .00 \\
\hline & 11 & - & .00 & - & - & .00 & .00 & .00 & .00 & .00 & .00 & .00 & .00 \\
\hline & 12 & - & .00 & - & .00 & .00 & .00 & .00 & .00 & .00 & .00 & .00 & .00 \\
\hline & 13 & - & .00 & - & .00 & .00 & .00 & .00 & .00 & .00 & .00 & .00 & .00 \\
\hline & 14 & - & .00 & - & .00 & .00 & .00 & .00 & .00 & .00 & .00 & .00 & .00 \\
\hline & 15 & - & .00 & - & .00 & .00 & .00 & .00 & .00 & .00 & .00 & .00 & .00 \\
\hline & 16 & - & .00 & - & .00 & .00 & .00 & .00 & .00 & .00 & .00 & .00 & .00 \\
\hline & 17 & - & .00 & - & .00 & .00 & .00 & .00 & .00 & .00 & .00 & .00 & .00 \\
\hline & 18 & - & .00 & - & .00 & .00 & .00 & .00 & .00 & .00 & .00 & .00 & .00 \\
\hline & 19 & - & .00 & - & .00 & .00 & .00 & .00 & .00 & .00 & .00 & .00 & .00 \\
\hline & 20 & - & .00 & - & .00 & .00 & .00 & .00 & .00 & .00 & .00 & .00 & .00 \\
\hline & 21 & - & .00 & - & .00 & .00 & .00 & .00 & .00 & .00 & .00 & .00 & .00 \\
\hline & 22 & - & .00 & - & .00 & .00 & .00 & .00 & .00 & .00 & .00 & .00 & .00 \\
\hline & 23 & .00 & .00 & - & .00 & .00 & .00 & .00 & .00 & .00 & .00 & .00 & .00 \\
\hline & 24 & .00 & .00 & - & .00 & .00 & .00 & .00 & .00 & .00 & .00 & .00 & .00 \\
\hline & 25 & .00 & .00 & - & .00 & .00 & .00 & .00 & .00 & .00 & .00 & .00 & .00 \\
\hline & 26 & .00 & .00 & - & .00 & .00 & .00 & .00 & .00 & .00 & .00 & .00 & .00 \\
\hline & 27 & .00 & .00 & - & .00 & .00 & .00 & .00 & .00 & .00 & .00 & .00 & .00 \\
\hline & 28 & .00 & .00 & - & .00 & .00 & .00 & .00 & .00 & .00 & .00 & .00 & .00 \\
\hline & 29 & .00 & .00 & - & .00 & .00 & .00 & .00 & .00 & .00 & .00 & .00 & .00 \\
\hline & 30 & .00 & - & - & .00 & .00 & .00 & .00 & .00 & .00 & .00 & .00 & .00 \\
\hline & 31 & .00 & - & - & - & .00 & - & .00 & .00 & - & .00 & - & .00 \\
\hline Total & & - & .00 & - & - & .00 & .00 & .00 & .00 & .00 & .00 & .00 & .00 \\
\hline Mean & & - & .00 & - & - & .00 & .00 & .00 & .00 & .00 & .00 & .00 & .00 \\
\hline Maximum & & - & .00 & - & - & .00 & .00 & .00 & .00 & .00 & .00 & .00 & .00 \\
\hline Minimum & & - & .00 & - & - & .00 & .00 & .00 & .00 & .00 & .00 & .00 & .00 \\
\hline \multicolumn{14}{|l|}{1985} \\
\hline & 1 & .00 & .00 & 0.00 & .00 & .00 & .00 & .00 & .00 & .27 & .00 & .01 & .00 \\
\hline & 2 & .00 & .00 & .00 & .00 & .00 & .00 & .00 & .00 & .02 & .00 & .00 & .00 \\
\hline & 3 & .00 & .00 & .00 & .00 & .00 & .00 & .00 & .00 & .00 & .03 & .00 & .00 \\
\hline & 4 & .00 & .00 & .00 & .00 & .00 & .00 & .00 & .00 & .00 & .01 & .00 & .00 \\
\hline & 5 & .00 & .00 & .00 & .00 & .00 & .00 & .00 & .00 & .00 & .00 & .00 & .00 \\
\hline
\end{tabular}


Table 3. Daily discharge at transect B, Big Four mine, August 1982 through September 1986-Continued

Month

\begin{tabular}{|c|c|c|c|c|c|c|c|c|c|c|c|c|}
\hline \multirow[b]{2}{*}{ Day } & \\
\hline & Jan. & Feb. & Mar. & Apr. & May & June & July & Aug. & Sept. & Oct. & Nov. & Dec. \\
\hline \multicolumn{13}{|l|}{ 1985-Continued } \\
\hline 6 & 0.00 & 0.00 & 0.00 & 0.00 & 0.00 & 0.00 & 0.00 & 0.00 & 0.00 & 0.00 & 0.00 & 0.00 \\
\hline 7 & .00 & .00 & .00 & .00 & .00 & .00 & .00 & .00 & .00 & .00 & .00 & .00 \\
\hline 8 & .00 & .00 & .00 & .00 & .00 & .00 & .00 & .00 & .00 & .00 & .00 & .00 \\
\hline 9 & .00 & .00 & .00 & .00 & .00 & .00 & .00 & .00 & .00 & .00 & .00 & .00 \\
\hline 10 & .00 & .00 & .00 & .00 & .00 & .00 & .00 & .00 & .00 & .00 & .00 & .00 \\
\hline 11 & .00 & .00 & .00 & .00 & .00 & .00 & .00 & .00 & .00 & .00 & .00 & .00 \\
\hline 12 & .00 & .00 & .00 & .00 & .00 & .00 & .00 & .00 & .00 & .00 & .00 & .00 \\
\hline 13 & .00 & .00 & .00 & .00 & .00 & .00 & .00 & .00 & .00 & .00 & .00 & .00 \\
\hline 14 & .00 & .00 & .00 & .00 & .00 & .00 & .00 & .00 & .00 & .00 & .00 & .00 \\
\hline 15 & .00 & .00 & .00 & .00 & .00 & .00 & .00 & .00 & .00 & .00 & .00 & .00 \\
\hline 16 & .00 & .00 & .00 & .00 & .00 & .00 & .00 & .00 & .00 & .00 & .00 & .00 \\
\hline 17 & .00 & .00 & .00 & .00 & .00 & .00 & .00 & .00 & .00 & .00 & .00 & .00 \\
\hline 18 & .00 & .00 & .00 & .00 & .00 & .00 & .00 & .00 & .00 & .00 & .00 & .00 \\
\hline 19 & .00 & .00 & .00 & .00 & .00 & .00 & .00 & .00 & .00 & .00 & .00 & .00 \\
\hline 20 & .00 & .00 & .00 & .00 & .00 & .00 & .00 & .00 & .00 & .00 & .00 & .00 \\
\hline 21 & .00 & .00 & .00 & .00 & .00 & .00 & .00 & .00 & .00 & .00 & .00 & .00 \\
\hline 22 & .00 & .00 & .00 & .00 & .00 & .00 & .00 & .00 & .00 & .00 & .00 & .00 \\
\hline 23 & .00 & .00 & .00 & .00 & .00 & .00 & .00 & .00 & .00 & .00 & .00 & .00 \\
\hline 24 & .00 & .00 & .00 & .00 & .00 & .00 & .00 & .00 & .00 & .00 & .00 & .00 \\
\hline 25 & .00 & .00 & .00 & .00 & .00 & .00 & .00 & .00 & .00 & .00 & .00 & .00 \\
\hline 26 & .00 & .00 & .00 & .00 & .00 & .00 & .00 & .00 & .00 & .00 & .00 & .00 \\
\hline 27 & .00 & .00 & .00 & .00 & .00 & .00 & .00 & .00 & .00 & .00 & .00 & .00 \\
\hline 28 & .00 & .00 & .00 & .00 & .00 & .00 & .00 & .00 & .00 & .00 & .00 & .00 \\
\hline 29 & .00 & - & .00 & .00 & .00 & .00 & .00 & .00 & .00 & .00 & .00 & .00 \\
\hline 30 & .00 & - & .00 & .00 & .00 & .00 & .00 & .00 & .00 & .00 & .00 & .00 \\
\hline 31 & .00 & - & .00 & - & .00 & - & .00 & .00 & - & .00 & - & .00 \\
\hline Total & .00 & .00 & .00 & .00 & .00 & .00 & .00 & .00 & .29 & .04 & .01 & .00 \\
\hline Mean & .00 & .00 & .00 & .00 & .00 & .00 & .00 & .00 & .01 & .01 & .00 & .00 \\
\hline Maximum & .00 & .00 & .00 & .00 & .00 & .00 & .00 & .00 & .27 & .03 & .01 & .00 \\
\hline Minimum & .00 & .00 & .00 & .00 & .00 & .00 & .00 & .00 & .00 & .00 & .00 & .00 \\
\hline
\end{tabular}

1986

\begin{tabular}{|c|c|c|c|c|c|c|c|c|c|c|c|c|}
\hline 1 & .06 & .01 & .00 & .00 & .00 & .00 & .00 & .01 & .00 & - & - & - \\
\hline 2 & .05 & .01 & .00 & .00 & .00 & .00 & .00 & .00 & .00 & - & - & - \\
\hline 3 & .04 & .02 & .00 & .00 & .00 & .00 & .00 & .01 & .00 & - & - & - \\
\hline 4 & .04 & .03 & .00 & .00 & .00 & .00 & .00 & .00 & .00 & - & - & - \\
\hline 5 & .08 & .03 & .01 & .00 & .00 & .00 & .00 & .01 & .00 & - & - & - \\
\hline 6 & .08 & .03 & .01 & .00 & .00 & .00 & .00 & .02 & .00 & - & - & - \\
\hline 7 & .07 & .03 & .01 & .00 & .00 & .00 & .00 & .00 & .00 & - & - & - \\
\hline 8 & .07 & .14 & .01 & .00 & .00 & .00 & .00 & .04 & .03 & - & - & - \\
\hline 9 & .07 & .11 & .01 & .00 & .00 & .00 & .00 & .05 & .00 & - & - & - \\
\hline 10 & .17 & .07 & .01 & .00 & .00 & .00 & .00 & .00 & .00 & - & - & - \\
\hline 11 & .10 &.$\infty 9$ & .03 & .00 & .00 & .00 & .00 & .00 & .00 & - & - & - \\
\hline 12 & .07 & .08 & .03 & .00 & .00 & .02 & .01 & .01 & .00 & - & - & - \\
\hline 13 & .06 & .05 & .03 & .00 & .00 & .09 & .01 & .02 & .00 & - & - & - \\
\hline 14 & .05 & .03 & .07 & .00 & .00 & .10 & .00 & .02 & .00 & - & - & - \\
\hline 15 & .04 & .03 & .34 & .00 & .00 & .10 & .00 & .00 & .00 & - & - & - \\
\hline
\end{tabular}


Table 3. Daily discharge at transect B, Big Four mine, August 1982 through September 1986-Continued

\begin{tabular}{|c|c|c|c|c|c|c|c|c|c|c|c|c|}
\hline \multirow[b]{2}{*}{ Day } & \multicolumn{12}{|c|}{ Month } \\
\hline & Jan. & Feb. & Mar. & Apr. & May & June & July & Aug. & Sept. & Oct. & Nov: & Dec. \\
\hline \multicolumn{13}{|l|}{ 1986-Continued } \\
\hline 17 & .03 & .01 & .16 & .00 & .00 & .04 & .00 & .00 & .00 & - & - & - \\
\hline 18 & .03 & .01 & .09 & .00 & .00 & .02 & .00 & .00 & .00 & - & - & - \\
\hline 19 & .03 & .01 & .07 & .00 & .00 & .01 & .00 & .00 & .00 & - & - & - \\
\hline 21 & .03 & .00 & .04 & .00 & .00 & .00 & .00 & .03 & .00 & - & - & - \\
\hline 22 & .03 & .00 & .03 & .00 & .00 & .13 & .00 & .03 & .00 & - & - & - \\
\hline 23 & .03 & .05 & .01 & .00 & .00 & .18 & .00 & .00 & .00 & - & - & - \\
\hline 24 & .03 & .05 & .00 & .00 & .00 & .06 & .00 & .02 & .00 & - & - & - \\
\hline 25 & .03 & .02 & .00 & .00 & .00 & .02 & .00 & .04 & .00 & - & - & - \\
\hline 29 & .03 & - & .00 & .00 & .00 & .00 & .00 & .03 & .00 & - & - & - \\
\hline 30 & .03 & - & .00 & .00 & .00 & .00 & .00 & .00 & .00 & - & - & - \\
\hline 31 & .02 & - & .00 & - & .00 & - & .03 & .00 & - & - & - & - \\
\hline Total & 1.53 & .96 & 1.91 & .00 & .00 & .82 & .09 & .65 & .03 & - & - & - \\
\hline Mean & .05 & .03 & .06 & .00 & .00 & .03 & .00 & .02 & .00 & - & - & - \\
\hline Maximum & .17 & .14 & .90 & .00 & .00 & .18 & .03 & .16 & .03 & - & - & - \\
\hline Minimum & .02 & .00 & .00 & .00 & .00 & .00 & .00 & .00 & .00 & - & - & - \\
\hline
\end{tabular}
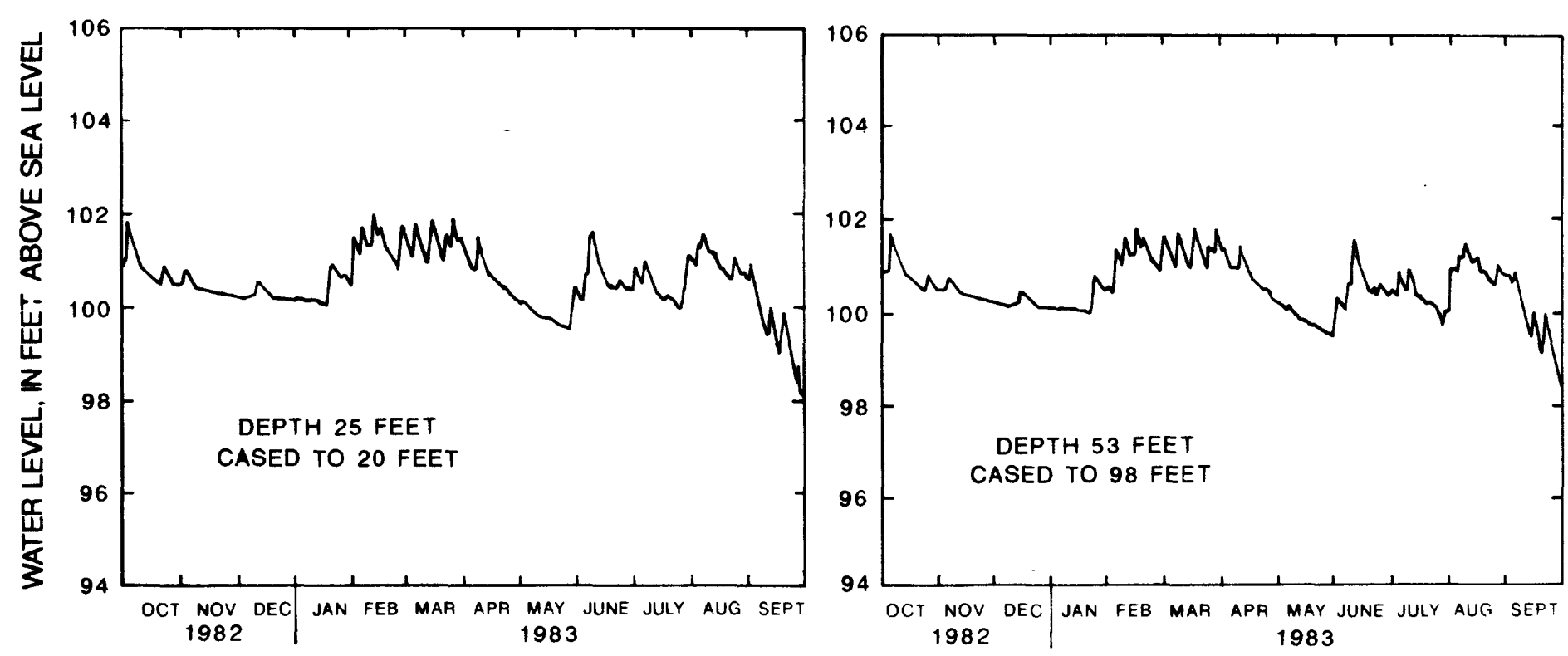

Figure 5. Water levels in well MM1, surficial aquifer, Big Four mine. Figure 6. Water levels in well MM3, intermediate aquifer, Big Four mine. 
All wells were destroyed during mining except MM4, MM5, and MM6 near transect C. Five wells in the surficial aquifer system, MS5, MS6, MS7, MS8, and MS9 (fig. 7), were installed in July 1985 following regrading of the 16 acres that were formerly wetlands. Periodic water-level measurements for selected wells at the test site for premining and postmining conditions are given in table 4 .

\section{Lithologic Data}

Two continuous cores were collected at the north end of transects $A$ and $C$ (fig. 3). Core number 1 near transect $A$ was 49 feet deep and core number 2 near transect $C$ was 55 feet deep. Lithologic descriptions of these cores are given in tables 5 and 6.

\section{Soils}

The upland soils adjacent to the wetlands generally consist of Ona fine sand and Pomello fine sand, and the wetlands soils consist of very fine alluvium and Rutlege soil (W.M. Saalman, U.S. Soil Conservation Service, written commun., 1983). Soil samples were collected from the north end of transects A, B, and C. A total of 13 samples was collected from six points at various depths up to 4 feet so that all major soil horizons at the site were sampled. Samples were analyzed by a private laboratory, generally using methods set forth by Jackson (1975). A summary of the soils analyses and the major chemical constituents and physical properties of the 13 samples are presented in table 7.

\section{Water Quality}

Water samples from the stream at transect $B$ and ground-water samples from shallow wells MM2 and MM6 were collected for analysis on November 17, 1982, and September 23, 1983, to define premining water quality. Samples also were collected after mining at well MS8 on August 28, 1985, and site B on August 25, 1986. Results of the analyses are presented in table 8 .

\section{Biology}

Floral and benthic invertebrate sampling was conducted at transects A, B, and C (fig. 3). Transects A and B had previously been sampled by Biological Research Associates (1982). Their floral sampling quadrats, 2 square meters in size, were sampled or visually checked at transects $A$ and B in January and June 1983, and new data were collected visually from transect C in January 1983. Transects B and C were sampled following mining in 1984, 1985, and 1986. Percentage of coverages were measured with a coarse grid or estimated. Results are presented in tables 9 and 10 . Benthic invertebrate samples were collected using a dip net sampler in the stream at the three transects in January and June 1983. Results of the benthic invertebrate dip-net survey are presented in table 11.

\section{Lonesome Mine Control Site}

The control basin is at the Lonesome mine, approximately 5 miles southwest of the test basin (fig. 2). The control basin monitor network, consisting of a stream gage and nine surficial aquifer wells, was established between July 1983 and January 1984. A botanical transect with four quadrats and three benthic invertebrate sampling sites was established in April 1984. The drainage area of the control basin is larger than the test basin, but the areas of hydric vegetation and hardwood forest are less than the test basin (10 acres versus 26 acres). Locations of the hydrologic and biologic monitoring sites are shown in figure 8.

\section{Streamflow}

Continuous gage heights and periodic discharge measurements were made on the unnamed tributary to the Little Manatee River. Daily discharge for the period of record, July 1983 through September 1986, is given in table 12 . There is no flow at the gage for several weeks during the dry season each year. Maximum instantaneous discharge for the period of record was $38 \mathrm{ft}^{3} / \mathrm{s}$ on September 1, 1985.

\section{Ground-Water Levels}

The network of nine shallow wells (ranging in depth from 7 to 9 feet) was established in January 1984. During dry periods, water levels in the surficial aquifer generally ranged from 3 to 4 feet below land surface (fig. 9). The maximum depth below land surface of 4.5 feet was observed on May 31, 1985, at well CO-3 (fig. 9). Observed water levels for the period January 1984 through October 1986 are shown in table 13. During wet periods, water levels range from at or near land surface to 0.5 foot below land surface. These levels are similar to levels observed at the test site.

\section{Water Quality}

Samples for water-quality analysis of the unnamed tributary at the control site were collected on September 23, 1983. Results of the analyses are given in table 14. 


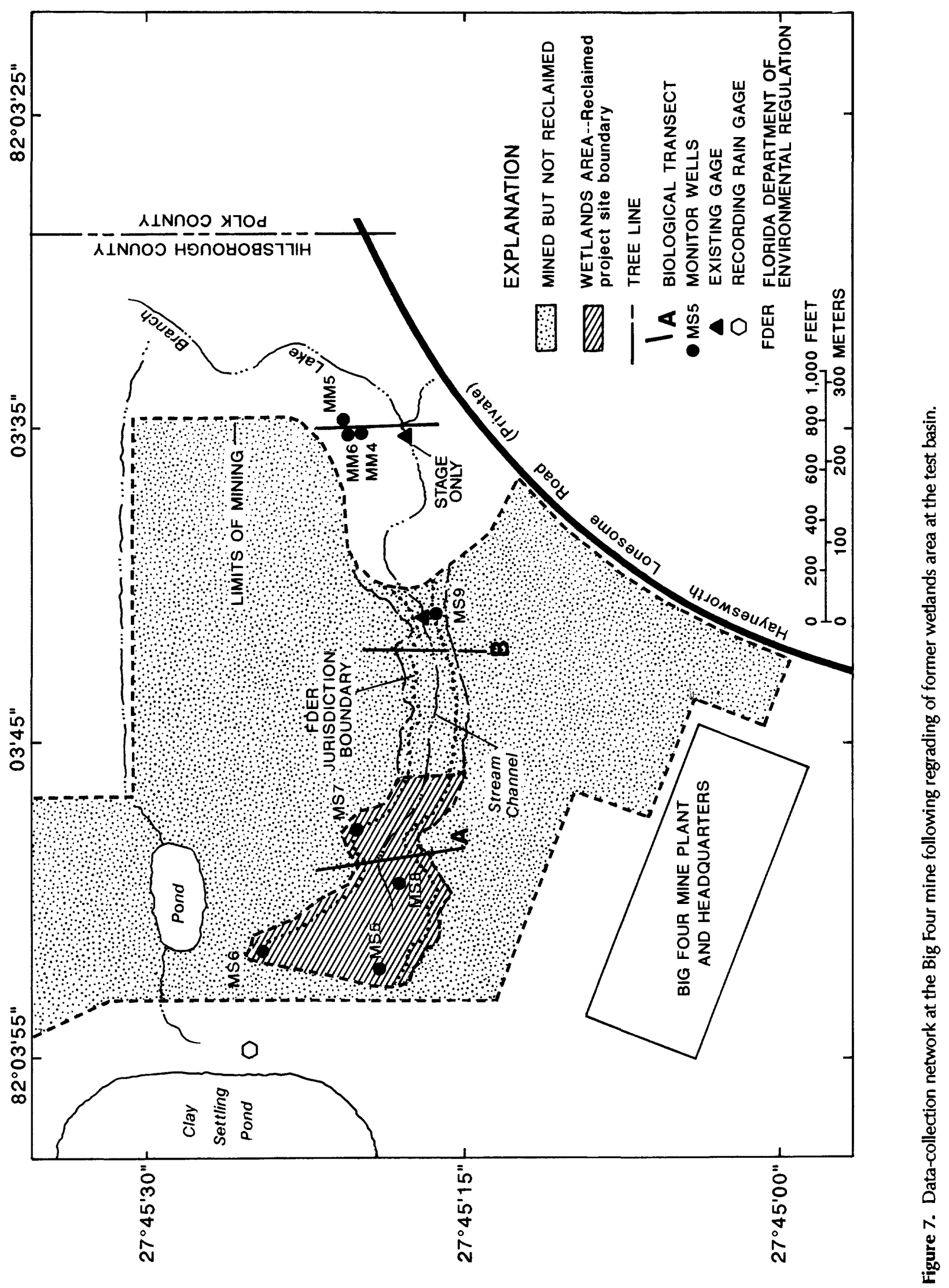


Table 4. Water-level observations at selected wells in the surficial aquifer system at Big Four mine

[Values are in feet above sea level. - $\rightarrow$, no data]

Well:

Depth of well (feet):

$\begin{array}{rrrrrrrrr}\text { MS1 } & \text { MS2 } & \text { MS3 } & \text { MS4 } & \text { MS5 } & \text { MS6 } & \text { MS7 } & \text { MS8 } & \text { MS9 } \\ 24.0 & 25.0 & 24.0 & 27.0 & 16.8 & 17.1 & 13.7 & 16.7 & 16.5 \\ 23.6 & 24.7 & 23.9 & 26.6 & 7.8 & 8.1 & 4.7 & 7.7 & 8.1\end{array}$

\begin{tabular}{|c|c|c|c|c|}
\hline \multirow[b]{2}{*}{ Date } & \multicolumn{4}{|c|}{ Premining water levels } \\
\hline & MS1 & MS2 & MS3 & MS4 \\
\hline $6-24-82$ & 103.53 & - & - & - \\
\hline $7-28-82$ & 103.14 & 103.02 & 101.51 & 96.97 \\
\hline $8-25-82$ & 102.90 & 102.83 & 101.97 & 96.89 \\
\hline $9-28-82$ & 103.47 & 103.06 & 102.53 & 97.02 \\
\hline $10-31-82$ & 101.80 & 102.73 & 100.65 & 96.70 \\
\hline $11-30-82$ & 99.99 & 102.01 & 99.37 & 96.05 \\
\hline $12-12-82$ & 99.50 & 101.90 & 99.01 & 95.63 \\
\hline $1-06-83$ & 99.00 & 101.79 & 98.73 & 95.37 \\
\hline $2-14-83$ & 103.39 & 103.08 & 102.76 & 97.01 \\
\hline $3-14-83$ & 102.56 & 102.73 & 101.96 & 96.69 \\
\hline $4-13-83$ & 102.77 & 102.67 & 101.96 & 96.79 \\
\hline $5-12-83$ & 100.44 & 101.50 & 99.33 & 95.63 \\
\hline $6-14-83$ & 102.17 & 102.79 & 101.19 & 96.23 \\
\hline 7-13-83 & 101.36 & 102.81 & 99.77 & 95.54 \\
\hline $8-11-83$ & 102.16 & 102.92 & 100.79 & 96.61 \\
\hline $9.23-83$ & 103.26 & 102.97 & 100.78 & 96.60 \\
\hline
\end{tabular}

\begin{tabular}{|c|c|c|c|c|c|}
\hline \multirow[b]{2}{*}{ Date } & \multicolumn{5}{|c|}{ Postmining water levels } \\
\hline & MS5 & MS6 & MS7 & MS8 & MS9 \\
\hline $7-28-85$ & 92.75 & 93.62 & 94.15 & 92.71 & 91.13 \\
\hline $8-28-85$ & 92.75 & 93.62 & 94.15 & 92.71 & 90.68 \\
\hline $9-30-85$ & 94.28 & 94.77 & 95.26 & 94.07 & 91.62 \\
\hline $10-28-85$ & 94.59 & 94.90 & 95.40 & 94.46 & 91.66 \\
\hline $11-22-85$ & 94.04 & 94.35 & 94.96 & 93.94 & 91.70 \\
\hline $12-18-85$ & 93.90 & 94.18 & 94.60 & 93.83 & 91.76 \\
\hline $1-31-86$ & 94.28 & 94.44 & 94.79 & 94.03 & 91.87 \\
\hline $2-27-86$ & 94.28 & 94.31 & 95.02 & 93.95 & 91.89 \\
\hline $3-24-86$ & 94.28 & 94.23 & 95.02 & 93.98 & 91.88 \\
\hline $4-28-86$ & 94.28 & 94.85 & 95.02 & 94.45 & 91.24 \\
\hline $5-29-86$ & 94.28 & 94.10 & 95.02 & 93.78 & 90.32 \\
\hline $6-26-86$ & 94.31 & 94.53 & 96.36 & 94.34 & 91.86 \\
\hline $7-30-86$ & 94.99 & 94.97 & 97.03 & 94.70 & 91.84 \\
\hline $8-20-86$ & 94.92 & 94.82 & 97.09 & 94.75 & 91.91 \\
\hline $10-09-86$ & 94.83 & 94.83 & 95.87 & 94.49 & 91.38 \\
\hline $11-12-86$ & 94.80 & 94.81 & 94.83 & 94.41 & 91.80 \\
\hline $12-16-86$ & 94.71 & 94.73 & 94.45 & 94.32 & 91.86 \\
\hline $1-14-87$ & 94.84 & 94.85 & 95.72 & 94.69 & 91.98 \\
\hline $2-10-87$ & 94.47 & 94.57 & 95.15 & 94.30 & 91.96 \\
\hline $3-09-87$ & 94.97 & 94.99 & 95.58 & 94.83 & 91.96 \\
\hline $4-09-87$ & 95.26 & 95.13 & 97.08 & 95.28 & 91.94 \\
\hline
\end{tabular}


Table 5. Lithologic description of core 1 at transect $A$, Big Four mine

\begin{tabular}{|c|c|c|}
\hline Lithologic description & $\begin{array}{l}\text { Thickness } \\
\text { (feet) }\end{array}$ & $\begin{array}{l}\text { Depth } \\
\text { (feet) }\end{array}$ \\
\hline Sand, fine to very fine quartz, light brown; organic debris and roots from 0 to 2 feet. & 5 & 5 \\
\hline $\begin{array}{l}\text { Same as above, varies in color from tan to light brown to dark brown throughout } \\
\text { section. }\end{array}$ & 15 & 20 \\
\hline Sand, fine quartz, brown. & 3.5 & 23.5 \\
\hline $\begin{array}{l}\text { Sand, fine quartz, tan to gray; with fine phosphate grains; some clay; fine to } \\
\text { gravelly black phosphate grains at } 36 \text { feet. }\end{array}$ & 12.5 & 36 \\
\hline No data. & 4 & 40 \\
\hline $\begin{array}{l}\text { Clay, light gray to light green and rust colored; } 2 \text { to } 3 \text { percent fine to very fine } \\
\text { quartz grains, grading to light gray with greater than } 5 \text { percent fine, to medium } \\
\text { quartz grains with some large mature quartz grains about } 43 \text { to } 44.5 \text { feet; grading } \\
\text { into limestone at } 44.5 \text { feet. }\end{array}$ & 4.5 & 44.5 \\
\hline $\begin{array}{l}\text { Limestone, cream, friable; greater than } 5 \text { percent fine to very fine mature } \\
\text { quartz grains, no fossils. }\end{array}$ & .5 & 45 \\
\hline Same as above; 5 percent quartz with some large grains. & 4 & 49 \\
\hline
\end{tabular}

Table 6. Lithologic description of core 2 at transect C, Big Four mine

\begin{tabular}{|c|c|c|}
\hline Lithologic description & $\begin{array}{l}\text { Thickness } \\
\text { (feet) }\end{array}$ & $\begin{array}{l}\text { Depth } \\
\text { (feet) }\end{array}$ \\
\hline Sand, fine quartz; gray becoming light tan at 5 feet; organic debris 0 to 1 foot. & 5 & 5 \\
\hline No data. & 2 & 7 \\
\hline Sand, fine quartz, gray. & 1 & 8 \\
\hline Sand; some clay. & 1.5 & 9.5 \\
\hline Clay, sandy, mottled rust and tan colored; with some small phosphate grains. & 4 & 13.5 \\
\hline Sand, fine quartz; some clay, loosely consolidated, light tan. & 6.5 & 20 \\
\hline $\begin{array}{l}\text { Sand, fine to very fine quartz, poorly sorted, loosely consolidated, gray; fine to } \\
\text { very fine to large quartz grains grading to gravel size with depth to about } 28 \text { feet } \\
\text { some phosphate grains; clay and some carbonates in matrix from } 28 \text { to } 31 \text { feet, } \\
\text { grading into limestone at } 31.5 \text { feet. }\end{array}$ & 11.5 & 31.5 \\
\hline $\begin{array}{l}\text { Limestone, cream, friable and loosely consolidated; fine to very fine quartz; } \\
\text { black phosphate grains; becoming more consolidated with depth. }\end{array}$ & 2 & 33.5 \\
\hline $\begin{array}{l}\text { Sand and calcareous clay, light gray to cream; fine to very fine quartz; phosphate } \\
\text { grains with some large gravel size black phosphate at } 36 \text { to } 37.5 \text { feet. }\end{array}$ & 4 & 37.5 \\
\hline Same as 31.5- to 33.5-foot description. & 2.5 & 40 \\
\hline $\begin{array}{l}\text { Limestone, soft, white cream; } 10 \text { percent fine to very fine quartz and black } \\
\text { phosphate grains; some small pelecypod molds. }\end{array}$ & 10 & 50 \\
\hline Same as above, tan; some clay, no observable fossils or molds. & 5 & 55 \\
\hline
\end{tabular}




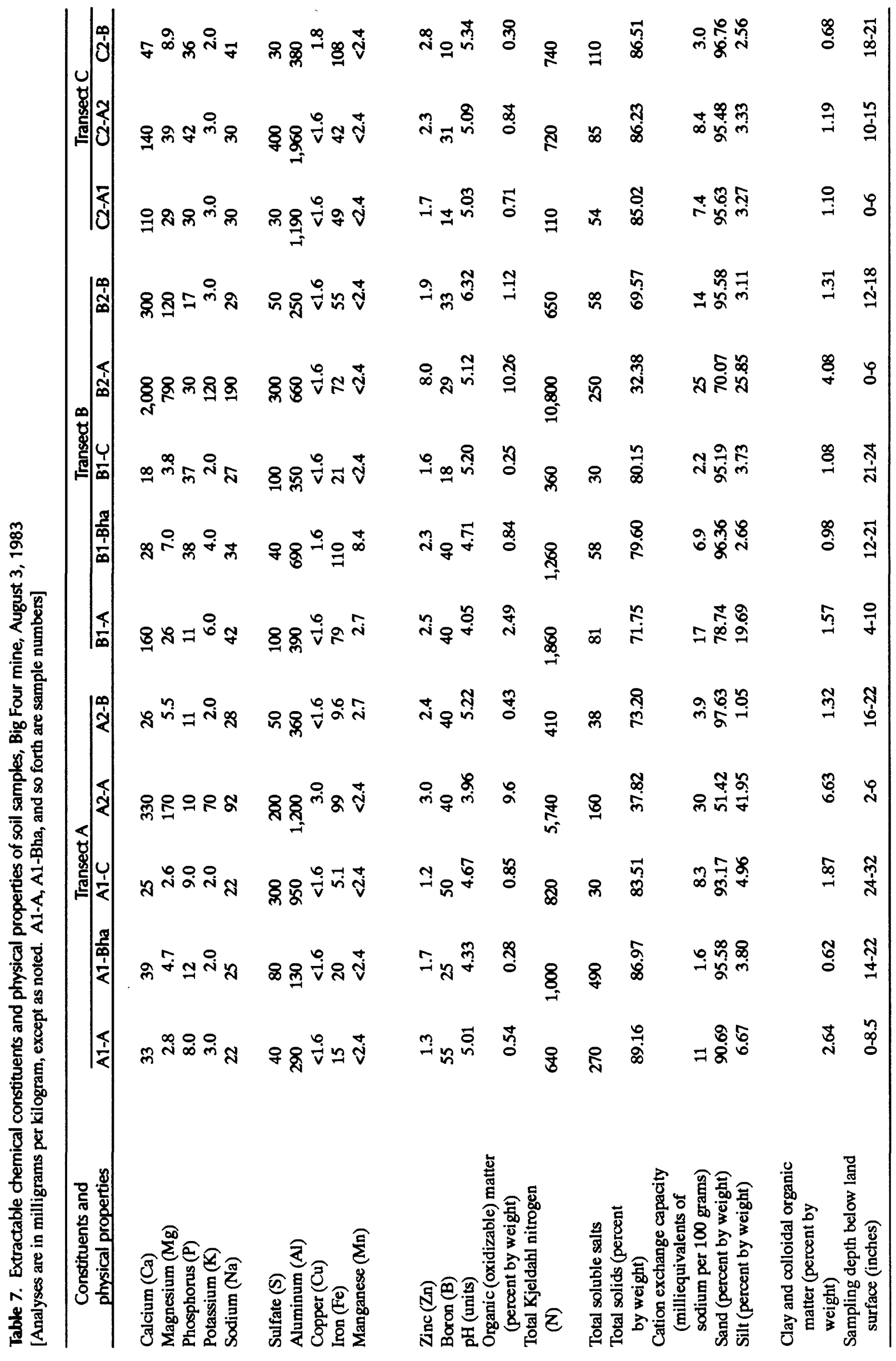


Table 8. Water-quality data from selected wells and a stream at Big Four mine

$\left[{ }^{\circ} \mathrm{C}\right.$, degrees Celsius; $\mathrm{ft}^{3} / \mathrm{s}$, cubic feet per second; NTU, nephelometric turbidity units; $\mu \mathrm{S} / \mathrm{cm}$, microsiemens per centimeter;

$\mathrm{mg} /$, milligrams per liter, $\mu \mathrm{g} /$, micrograms per liter; $\mathrm{pCi} / \mathrm{L}$, picocuries per liter, —, no data]

\begin{tabular}{|c|c|c|c|c|c|c|c|c|}
\hline \multirow{2}{*}{$\begin{array}{l}\text { Constituents and } \\
\text { physical properties }\end{array}$} & \multicolumn{2}{|c|}{$\begin{array}{c}\text { Well MM6 } \\
(274517082032901) \\
\end{array}$} & \multicolumn{3}{|c|}{$\begin{array}{c}\text { Tributary at B } \\
(274517082034100)\end{array}$} & \multirow{2}{*}{$\begin{array}{c}\text { Well MM8 } \\
\frac{(274518082035201)}{8-28-85} \\
\end{array}$} & \multicolumn{2}{|c|}{$\begin{array}{c}\text { Well MM2 } \\
(274520082035101)\end{array}$} \\
\hline & $11-17-82$ & $9-23-83$ & $11-17-82$ & $9-23-83$ & $8-25-86$ & & $11-17-82$ & $9-23-83$ \\
\hline Water temperature $\left({ }^{\circ} \mathrm{C}\right)$ & 23.5 & - & 21.0 & - & 27.5 & 30.0 & 24.0 & - \\
\hline $\begin{array}{l}\text { Streamflow, instan- } \\
\text { taneous }\left(\mathrm{ft}^{3} / \mathbf{s}\right)\end{array}$ & - & - & 0.13 & 0.13 & 0.03 & - & - & - \\
\hline Turbidity (NTU) & 3.7 & - & 1.3 & - & - & - & 43 & - \\
\hline $\begin{array}{l}\text { Color (platinum- } \\
\text { cobalt units) } \\
\text { Specific conductance }\end{array}$ & 30 & - & 50 & - & 200 & 30 & 20 & - \\
\hline$(\mu \mathrm{S} / \mathrm{cm})$ & 355 & 367 & 100 & 102 & 175 & 302 & 98 & 93 \\
\hline $\begin{array}{l}\text { Oxygen, dissolved } \\
(\mathrm{mg} / \mathrm{L})\end{array}$ & - & - & 5.3 & - & - & - & - & - \\
\hline $\begin{array}{l}\text { Oxygen demand, chemi- } \\
\text { cal, high level }(\mathrm{mg} / \mathrm{L})\end{array}$ & 30 & - & 25 & - & - & - & 30 & - \\
\hline $\begin{array}{l}\text { pH (standard units) } \\
\text { pH, lab (standard }\end{array}$ & 7.40 & 6.20 & 6.00 & 4.90 & - & 5.20 & 4.90 & 4.60 \\
\hline $\begin{array}{l}\text { units) } \\
\text { Nitrogen, organic, }\end{array}$ & 7.30 & 7.30 & 6.20 & 5.50 & 5.50 & 5.30 & 5.50 & - \\
\hline total (mg/L as N) & - & - & 0.28 & - & 1.1 & - & - & 0.25 \\
\hline $\begin{array}{l}\text { Nitrogen, organic, } \\
\text { dissolved (mgl as } N \text { ) }\end{array}$ & 0.02 & - & - & - & - & - & 0.06 & - \\
\hline $\begin{array}{l}\text { Nitrogen, ammonia, } \\
\text { dissolved (mg/L as } N \text { ) }\end{array}$ & 0.220 & - & 0.020 & 0.040 & 0.070 & - & 0.130 & - \\
\hline $\begin{array}{l}\text { Nitrogen, ammonia, } \\
\text { total (mg/L as } \mathrm{N} \text { ) }\end{array}$ & - & 0.280 & 0.020 & - & 0.060 & - & - & 0.150 \\
\hline $\begin{array}{l}\text { Nitrogen, nitrite, } \\
\text { dissolved (mg/L as } \mathrm{N} \text { ) } \\
\text { Nitrogen, nitrite. }\end{array}$ & 0.00 & - & 0.00 & - & 0.010 & - & 0.00 & - \\
\hline $\begin{array}{l}\text { Nitrogen, nitrite, } \\
\text { total (mg/L as N) }\end{array}$ & - & 0.010 & - & - & - & - & - & - \\
\hline $\begin{array}{l}\text { Nitrogen, nitrate, } \\
\text { dissolved (mg/L as } N \text { ) }\end{array}$ & 0.00 & - & 0.030 & - & - & - & 0.00 & - \\
\hline $\begin{array}{l}\text { Nitrogen, ammonia plus } \\
\text { organic, dissolved } \\
\text { (mg/Las } \mathrm{N} \text { ) }\end{array}$ & 0.24 & - & - & - & - & - & 0.19 & - \\
\hline $\begin{array}{l}\text { Nitrogen, ammonia plus } \\
\text { organic, total (mg/L }\end{array}$ & & & & & & & & \\
\hline $\begin{array}{l}\text { as } \mathrm{N} \text { ) } \\
\text { Nitrogen, } \mathrm{NO}_{2}+\mathrm{NO}_{3} \text {, }\end{array}$ & - & - & 0.30 & 0.60 & 1.2 & - & - & 0.40 \\
\hline $\begin{array}{l}\text { total }(\mathrm{mg} / \mathrm{L} \text { as } \mathrm{N}) \\
\text { Nitrogen, } \mathrm{NO}_{2}+\mathrm{NO}_{3}\end{array}$ & - & $<0.100$ & - & - & - & - & - & - \\
\hline $\begin{array}{l}\text { ittrogen, } N_{2}+N_{3} \\
\text { dissolved }(m g / L \text { as } N)\end{array}$ & 0.00 & - & 0.030 & $<0.100$ & $<0.100$ & - & 0.00 & - \\
\hline $\begin{array}{l}\text { Phosphate, ortho, dis- } \\
\left.\text { solved (mg/L as } \mathrm{PO}_{4}\right) \\
\text { Phosphorus, total }\end{array}$ & 0.06 & - & 2.1 & 4.6 & 3.7 & - & 0.46 & - \\
\hline $\begin{array}{l}\text { (mg/L as } \mathrm{P} \text { ) } \\
\text { Phosphorus, dissolved }\end{array}$ & - & 0.140 & 0.740 & 1.70 & 2.00 & - & - & 0.300 \\
\hline $\begin{array}{l}\text { (mg/L as } \mathrm{P} \text { ) } \\
\text { Phosphorus, ortho, dis- }\end{array}$ & 0.020 & - & 0.680 & 1.50 & 1.90 & - & 0.160 & - \\
\hline $\begin{array}{l}\text { solved (mg/L as } \mathrm{P}) \\
\text { Carbon, organic, total }\end{array}$ & 0.020 & - & 0.680 & 1.50 & 1.20 & - & 0.150 & - \\
\hline (mg/Las $\mathrm{C}$ ) & 6.7 & - & 8.2 & - & 56 & - & 3.9 & - \\
\hline
\end{tabular}


Table 8. Water-quality data from selected wells and a stream at Big Four mine-Continued

\begin{tabular}{|c|c|c|c|c|c|c|c|c|}
\hline \multirow{2}{*}{$\begin{array}{l}\text { Constituents and } \\
\text { physical properties }\end{array}$} & \multicolumn{2}{|c|}{$\begin{array}{c}\text { Well MM6 } \\
(274517082032901)\end{array}$} & \multicolumn{3}{|c|}{$\begin{array}{c}\text { Tributary at B } \\
(274517082034100)\end{array}$} & \multirow{2}{*}{$\begin{array}{c}\text { Well MM8 } \\
\frac{(274518082035201)}{8-28-85}\end{array}$} & \multicolumn{2}{|c|}{$\begin{array}{c}\text { Well MM2 } \\
(274520082035101)\end{array}$} \\
\hline & $11-17-82$ & $9-23-83$ & $11-17-82$ & $9-23-83$ & $8-25-86$ & & $11-17-82$ & $9-23-83$ \\
\hline $\begin{array}{l}\text { Carbon, organic, dis- } \\
\text { solved (mg/L as C) }\end{array}$ & 4.6 & - & 8.2 & - & 42 & - & 3.9 & - \\
\hline Hardness (mg/L as & & & & & & & & \\
\hline $\left.\mathrm{CaCO}_{3}\right)$ & 170 & - & 32 & - & 45 & - & 22 & - \\
\hline $\begin{array}{l}\text { Calcium, dissolved } \\
\text { (mg/L as Ca) }\end{array}$ & 36 & - & 7.8 & - & 12 & - & 4.2 & - \\
\hline $\begin{array}{l}\text { Magnesium, dissolved } \\
(\mathrm{mg} / \mathrm{L} \text { as } \mathrm{Mg} \text { ) }\end{array}$ & 20 & - & 3.1 & - & 3.6 & - & 2.8 & - \\
\hline $\begin{array}{l}\text { Sodium, dissolved } \\
\text { (mg/L as Na) }\end{array}$ & 10 & - & 8.8 & - & 13 & - & 8.2 & - \\
\hline $\begin{array}{l}\text { Sodium adsoption } \\
\text { ratio }\end{array}$ & 0.3 & - & 0.7 & - & 0.9 & - & 0.8 & - \\
\hline Percent sodium & 11 & - & 37 & - & 38 & - & 45 & - \\
\hline $\begin{array}{l}\text { Potassium, dissolved } \\
\text { (mg/L as K) } \\
\text { Chloride, dissolved }\end{array}$ & 1.3 & - & 0.10 & - & 0.40 & - & 0.10 & - \\
\hline $\begin{array}{l}\text { (mg/L as } \mathrm{Cl} \text { ) } \\
\text { Sulfate dissolved }\end{array}$ & 8.8 & - & 12 & - & 21 & 11 & 13 & - \\
\hline $\begin{array}{l}\text { Sulfate, dissolved } \\
\text { (mg/L as } \mathrm{SO}_{4} \text { ) }\end{array}$ & 0.2 & - & 21 & - & 34 & 84 & 16 & - \\
\hline $\begin{array}{l}\text { Fluoride, dissolved } \\
(\mathrm{mg} / \mathrm{L} \text { as } \mathrm{F})\end{array}$ & 0.50 & - & 0.60 & - & 0.80 & 0.30 & 0.20 & - \\
\hline $\begin{array}{l}\text { Silica, dissolved } \\
\text { (mg/L as SiO2) }\end{array}$ & 19 & - & 6.8 & - & 11 & - & 5.3 & - \\
\hline $\begin{array}{l}\text { Arsenic, dissolved } \\
\text { ( } \mu \mathrm{g} / \mathrm{L} \text { as As) } \\
\text { Boron, dissolved }\end{array}$ & - & - & - & - & - & 7 & - & - \\
\hline $\begin{array}{l}\text { ( } \mu \mathrm{g} / \mathrm{L} \text { as } \mathrm{B}) \\
\text { Cadmium, dissolved }\end{array}$ & 30 & 40 & 60 & 80 & 20 & 30 & 20 & - \\
\hline$(\mu \mathrm{g} / \mathrm{L}$ as $\mathrm{Cd})$ & $<1$ & 1 & $<1$ & 2 & $<1$ & $<1$ & $<1$ & - \\
\hline $\begin{array}{l}\text { Chromium, dissolved } \\
(\mu \mathrm{g} / \mathrm{L} \text { as } \mathrm{Cr})\end{array}$ & 10 & 10 & 10 & $<10$ & $<10$ & $<10$ & 10 & - \\
\hline $\begin{array}{l}\text { Copper, dissolved } \\
(\mu \mathrm{g} / \mathrm{L} \text { as } \mathrm{Cu}) \\
\text { Iron, dissolved }\end{array}$ & $<1$ & 1 & 1 & 1 & $<3$ & 2 & $<1$ & - \\
\hline $\begin{array}{l}(\mu \mathrm{g} / \mathrm{L} \text { as } \mathrm{Fe}) \\
\text { Lead, dissolved }\end{array}$ & - & 650 & - & - & - & 3,400 & - & - \\
\hline $\begin{array}{l}(\mu \mathrm{g} / \mathrm{L} \text { as } \mathrm{Pb}) \\
\text { Manganese, dissolved }\end{array}$ & $<1$ & 2 & 2 & 4 & $<5$ & 2 & $<1$ & - \\
\hline$(\mu \mathrm{g} / \mathrm{L}$ as $\mathrm{Mn})$ & 29 & 19 & 6 & 5 & 6 & 25 & 4 & - \\
\hline $\begin{array}{l}\text { Molybdenum, dissolved } \\
\text { ( } \mathrm{g} / \mathrm{L} \text { as Mo) } \\
\text { Nickel, dissolved }\end{array}$ & $<1$ & $<1$ & 1 & $<1$ & $<1$ & 2 & 1 & - \\
\hline $\begin{array}{l}\text { Nickel, dissolved } \\
\text { ( } \mu \mathrm{g} / \mathrm{Las} \mathrm{Ni}) \\
\text { Strontium, dissolved }\end{array}$ & 2 & $<1$ & 1 & $<1$ & 2 & 7 & 1 & - \\
\hline $\begin{array}{l}\text { ( } \mu \mathrm{g} / \mathrm{L} \text { as } \mathrm{Sr} \text { ) } \\
\text { Vanadium, dissolved }\end{array}$ & 34 & - & 10 & - & 28 & - & 10 & - \\
\hline $\begin{array}{l}\text { ( } \mu g / L \text { as } V) \\
\text { Zinc, dissolved }\end{array}$ & 1 & $<1$ & 3 & 9 & 9 & $<6$ & 2 & - \\
\hline$(\mu g / L$ as $Z n)$ & $<4$ & $<3$ & $<4$ & 9 & 11 & 19 & 6 & - \\
\hline
\end{tabular}


Table 8. Water-quality data from selected wells and a stream at Big Four mine-Continued

\begin{tabular}{|c|c|c|c|c|c|c|c|c|}
\hline \multirow{2}{*}{$\begin{array}{l}\text { Constituents and } \\
\text { physical propenties }\end{array}$} & \multicolumn{2}{|c|}{$\begin{array}{c}\text { Well MM6 } \\
(274517082032901) \\
\end{array}$} & \multicolumn{3}{|c|}{$\begin{array}{c}\text { Tributary at B } \\
(274517082034100) \\
\end{array}$} & \multirow{2}{*}{$\begin{array}{c}\text { Well MM8 } \\
\frac{(274518082035201)}{8-28-85} \\
\end{array}$} & \multicolumn{2}{|c|}{$\begin{array}{c}\text { Well MM2 } \\
(274520082035101) \\
\end{array}$} \\
\hline & $11-17-82$ & $9-23-83$ & $11-17-82$ & $9-23-83$ & $8-25-86$ & & $11-17-82$ & $9-23-83$ \\
\hline $\begin{array}{l}\text { Aluminum, dissolved } \\
(\mu g / L \text { as } A) \\
\text { Gross beta, dissolved }\end{array}$ & $<10$ & 30 & 230 & 480 & 880 & 50 & 250 & - \\
\hline $\begin{array}{l}\text { (PCi/L as CS-137) } \\
\text { Gross beta, suspended, } \\
\text { total ( } \mathrm{pC} / \mathrm{L} / \mathrm{L} \text { as }\end{array}$ & $<2.8$ & $<3.1$ & 1.1 & 2.7 & - & - & 1.9 & 7.1 \\
\hline $\begin{array}{l}\text { CS-137) } \\
\text { Radium 226, dissolved, }\end{array}$ & $<0.4$ & - & $<0.4$ & - & - & - & 40 & - \\
\hline $\begin{array}{l}\text { radon method (pCi/L) } \\
\text { Uranium, natural, dis- }\end{array}$ & 0.74 & 1.3 & 0.14 & 0.22 & - & - & 1.8 & 0.67 \\
\hline $\operatorname{solved}(\mu g / L$ as $U)$ & 1.8 & - & 1.2 & - & - & - & 4.5 & - \\
\hline $\begin{array}{l}\text { Phenols, total }(\mu g / L) \\
\text { Solids, residue at } 180\end{array}$ & 4 & - & 4 & - & 2 & 6 & 6 & - \\
\hline${ }^{\circ} \mathrm{C}$, dissolved (mg/L) & 205 & - & 80 & - & 152 & - & 65 & - \\
\hline $\begin{array}{l}\text { Solids, dissolved } \\
\text { (tons per day) }\end{array}$ & 0.0 & - & 0.03 & - & 0.01 & - & 0.0 & - \\
\hline $\begin{array}{l}\text { Phosphorus, ortho, } \\
\text { total (mg/L as } P \text { ) } \\
\text { Acidity (mg/L as } \mathrm{H})\end{array}$ & $\underline{-}$ & 0.060 & $\overline{-}$ & - & $\overline{0.5}$ & $\overline{-}$ & - & $\overline{-}$ \\
\hline $\begin{array}{l}\text { Iodide, dissolved } \\
\text { (mg/L as I) } \\
\text { Bromide, dissolved }\end{array}$ & 0.010 & 0.005 & 0.010 & 0.004 & 0.012 & $<0.001$ & 0.040 & - \\
\hline $\begin{array}{l}\text { (mg/L as } \mathrm{Br} \text { ) } \\
\text { Mercury, dissolved }\end{array}$ & 0.20 & 0.030 & 0.19 & 0.15 & 0.11 & $<0.010$ & 0.17 & - \\
\hline $\begin{array}{l}\text { ( } \mu \mathrm{g} / \mathrm{L} \text { as } \mathrm{Hg}) \\
\text { Uranium, dissolved, }\end{array}$ & $<0.1$ & $<0.1$ & $<0.1$ & $<0.1$ & - & 0.1 & $<0.1$ & - \\
\hline $\begin{array}{l}\text { extraction ( } \mu g / L) \\
\text { Gross alpha, dissolved } \\
\text { ( } \mu g / L \text { as U-nat) }\end{array}$ & $<5.3$ & 0.09 & - & 0.19 & - & - & - & $\begin{array}{l}0.07 \\
15\end{array}$ \\
\hline $\begin{array}{l}\text { Gross al pha, suspended, } \\
\text { total ( } \mu g / L \text { as U-nat) }\end{array}$ & $<0.4$ & - & 1.8 & - & - & - & 120 & - \\
\hline $\begin{array}{l}\text { Gross beta, dissolved } \\
\text { (pCi/Las } \mathrm{Sr} / \mathrm{YT}-90)\end{array}$ & $<2.7$ & $<2.6$ & 1.1 & 2.3 & - & - & 1.8 & 5.9 \\
\hline $\begin{array}{l}\text { Gross beta, suspended, } \\
\text { total (pCi } / \mathrm{L} \text { as }\end{array}$ & & & & & & & & \\
\hline $\mathrm{Sr} / \mathrm{YT}-90)$ & $<0.4$ & - & $<0.4$ & - & - & - & 39 & - \\
\hline $\begin{array}{l}\text { Alkalinity, lab (mg/L } \\
\text { as CaCO3) } \\
\text { Bicarbonate, IT-lab }\end{array}$ & 182 & - & 7.0 & - & 11 & 19 & 3.0 & - \\
\hline $\begin{array}{l}(\mathrm{mg} / \mathrm{L} \text { as } \mathrm{HCO} 3) \\
\text { Carbonate, IT-lab }\end{array}$ & 222 & - & 9.0 & - & - & - & 4.0 & - \\
\hline (mg/L as $\left.\mathrm{CO}_{3}\right)$ & $\mathbf{0}$ & - & 0 & - & - & - & 0 & - \\
\hline
\end{tabular}


Table 9. Composition of flora at transect A, Big Four mine, 1983 $[-$, no data]

\begin{tabular}{ccc}
\hline & \multicolumn{2}{c}{ Percent coverage } \\
\cline { 2 - 3 } Species & January 1983 & June 1983 \\
\hline
\end{tabular}

Quadrat 1

Percent coverage

Acer rubrum

Red maple

Liquidambar styraciflua Sweet gum

Quercus nigra

Water oak

Osmunda cinnamomea

Cinnamon fern

Psilotum nudum

Whisk fern

Saururus cernuus

$$
\text { Lizard tail }
$$

Smilax walteri

Coral greenbrier

Toxicodendron radicans

Poison ivy

Woodwardia areolata

Chain fern

\section{5}

1

5

5

1

1

1

- 1

10

Woodwardia virginica

Chain fern

Litter and unvegetated

Quadrat 2

Acer rubrum

Red maple

Liquidambar styraciflua Sweet gum

Quercus nigra

Water oak

Rhododendron viscosum

Swamp azalea

Psilotum nudum

Whisk ferm

Smilax walteri

Coral greenbrier

Vitis rotundifolia

Muscadine grape
1

1

10

1

1

1

1
15

66
5

Quadrat 2-Continued

Woodwardia areolata

Chain fern

20

40

Litter and unvegetated

64

8

Quadrat 3

Quercus nigra

Water oak

Quercus pumila

Runner oak

January 1983

June 1983

Osmunda cinnamomea

Cinnamon fern

Cephalanthus occidentales

Buttonbush

Psilotum nudum

Whisk fern

Serenoa repens

Saw palmetto

Vitis rotundifolia

Muscadine grape

Woodwardia areolata

Chain fern

Litter and unvegetated

$\begin{array}{rr}1 & 1 \\ - & 1 \\ 5 & 10 \\ - & 1 \\ & \\ 1 & 5 \\ 20 & \\ & \\ 1 & 5 \\ 30 & 40 \\ 42 & 7\end{array}$

Quadrat 4

Quercus nigra

Water oak

Rhododendron viscosum

Swamp azalea

Paspalum sp.

Psilotum nudum

Whisk fern

Serenoa repens

Saw palmetto

5

Woodwardia areolata

Chain fern

$10 \quad 20$

110

1

10

15

15

Litter and unvegetated 
Table 10. Composition of flora at transects B and C, Big Four mine, 1983 through 1986

$[-$, no data $]$

\begin{tabular}{|c|c|c|c|c|c|c|}
\hline \multirow[b]{2}{*}{ Species } & \multicolumn{6}{|c|}{ Percent coverage } \\
\hline & $\begin{array}{c}\text { January } \\
1983\end{array}$ & $\begin{array}{l}\text { June } \\
1983 \\
\end{array}$ & $\begin{array}{c}\text { December } \\
1984 \\
\end{array}$ & $\begin{array}{l}\text { June } \\
1985 \\
\end{array}$ & $\begin{array}{c}\text { January } \\
1986 \\
\end{array}$ & $\begin{array}{l}\text { July } \\
198\end{array}$ \\
\hline \multicolumn{7}{|l|}{ Transect B, Quadrat 1} \\
\hline $\begin{array}{l}\text { Acer rubrum } \\
\text { Red maple } \\
\text { Osmunda cinnamomea }\end{array}$ & 1 & 1 & - & - & - & - \\
\hline $\begin{array}{l}\text { Cinnamon fern } \\
\text { Saururus cernuus }\end{array}$ & 10 & 15 & 10 & 15 & - & 10 \\
\hline $\begin{array}{l}\text { Lizard tail } \\
\text { Smilax sp. }\end{array}$ & 5 & 15 & 10 & 15 & - & 5 \\
\hline $\begin{array}{l}\text { Brier } \\
\text { Toxicodendron radicans }\end{array}$ & 1 & 1 & 10 & 10 & 1 & - \\
\hline $\begin{array}{c}\text { Poison ivy } \\
\text { Vitis rotundifolia }\end{array}$ & - & 1 & - & - & - & 1 \\
\hline $\begin{array}{c}\text { Muscadine grape } \\
\text { Woodwardia areolata }\end{array}$ & 1 & 5 & - & - & - & 1 \\
\hline Chain fern & 18 & 25 & $\begin{array}{l}20 \\
50\end{array}$ & 30 & $\begin{array}{l}10 \\
89\end{array}$ & $\begin{array}{l}50 \\
33\end{array}$ \\
\hline
\end{tabular}

Transect B, Quadrat 2

Acer rubrum

Red maple

Magnolia virginiana

Southem magnolia

Saururus cernuus

Lizard tail

Smilax walteri

Coral greenbrier

Toxicodendron radicans

Poison ivy

Woodwardia areolata

Chain fern

Litter and unvegetated

20
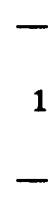

1

77

1

5

1

Whisk fern

Rubus trivialis

Blackberry

Smilax walteri Coral greenbrier

Vitis rotundifolia Muscadine grape

Saururus cernuus Lizard tail

Woodwardia areolata

Chain fern

Woodwardia virginica

Chain fern

Litter and unvegetated

Transect B, Quadrat 3

Red maple

smundo cinnamomea

1

5

15

$\overline{73}$

73
1

1

20

40

5

5

1

10

38

5

-

20

5

20

1

10

10

1

10

5

20

49

40

30

60

14

1

1

50

33

uly

86

1

1

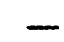

-

20

10

5

5

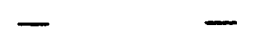

$-$

10

$\begin{array}{ll}- & - \\ - & -\end{array}$

-

$-$

1
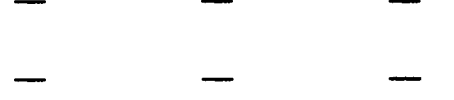

15

30

40

20

40

-

$-$

$-$

10

20

39

65

60

70

8 
Table 10. Composition of flora at transects B and C, Big Four mine, 1983 through 1986-Continued $[-$, no data $]$

\begin{tabular}{|c|c|c|c|c|c|c|}
\hline \multirow[b]{2}{*}{ Species } & \multicolumn{6}{|c|}{ Percent coverage } \\
\hline & $\begin{array}{c}\text { January } \\
1983\end{array}$ & $\begin{array}{l}\text { June } \\
1983 \\
\end{array}$ & $\begin{array}{c}\text { December } \\
1984 \\
\end{array}$ & $\begin{array}{l}\text { June } \\
1985 \\
\end{array}$ & $\begin{array}{c}\text { January } \\
1986 \\
\end{array}$ & $\begin{array}{c}\text { July } \\
1986 \\
\end{array}$ \\
\hline \multicolumn{7}{|l|}{ Transect B, Quadrat 4} \\
\hline $\begin{array}{l}\text { Acer rubrum } \\
\text { Red maple } \\
\text { Psilotum nudum }\end{array}$ & 1 & 1 & 1 & - & 10 & - \\
\hline $\begin{array}{l}\text { Whisk fern } \\
\text { Woodwardia areolata }\end{array}$ & - & 1 & - & - & - & 5 \\
\hline $\begin{array}{l}\text { Chain fern } \\
\text { Woodwardia virginica }\end{array}$ & 40 & 70 & 40 & 70 & 15 & 75 \\
\hline $\begin{array}{l}\text { Chain fern } \\
\text { Litter and unvegetated }\end{array}$ & $\overline{59}$ & $\overline{28}$ & $\overline{59}$ & $\overline{30}$ & $\overline{75}$ & $\begin{array}{l}10 \\
10\end{array}$ \\
\hline \multicolumn{7}{|l|}{ Transect C, Quadrat 1} \\
\hline $\begin{array}{l}\text { Sagittaria lancifolia } \\
\text { Arrowhead } \\
\text { Pontederia lanceolata }\end{array}$ & 20 & 15 & - & - & 10 & - \\
\hline $\begin{array}{c}\text { Pickerelweed } \\
\text { Ludwigia peruviana }\end{array}$ & 10 & 20 & - & - & 10 & - \\
\hline $\begin{array}{l}\text { Primrose willow } \\
\text { Ludwigia repens }\end{array}$ & 10 & 20 & - & - & 10 & - \\
\hline Red ludwigia & 30 & 40 & - & - & 30 & - \\
\hline Litter and unvegetated & 30 & 5 & - & - & 40 & - \\
\hline
\end{tabular}

Table 11. Benthic invertebrate dip-net survey at transects A, B, and C, Big Four mine $[-$, no data $]$

\begin{tabular}{|c|c|c|}
\hline Species & $\begin{array}{c}\text { January } 1983 \\
\text { count }\end{array}$ & $\begin{array}{c}\text { June } 1983 \\
\text { count } \\
\end{array}$ \\
\hline \multicolumn{3}{|l|}{ Transect A } \\
\hline \multicolumn{3}{|l|}{ Annelida } \\
\hline \multicolumn{3}{|l|}{ Hirudenea (leeches) } \\
\hline $\begin{array}{l}\text { Placosdella ornata } \\
\text { Arthropoda }\end{array}$ & 1 & - \\
\hline \multicolumn{3}{|l|}{ Crustacea } \\
\hline \multicolumn{3}{|l|}{ Amphipoda (scuds) } \\
\hline \multicolumn{2}{|l|}{ Decopoda (crayfish) } & 15 \\
\hline Procambaras sp. & 6 & - \\
\hline $\begin{array}{l}\text { Juvenile crayfish } \\
\text { Isopoda (sow-bugs) }\end{array}$ & Isopoda (sow-bugs) & - \\
\hline $\begin{array}{l}\text { Unidentifiable isopod } \\
\text { Insecta }\end{array}$ & - & 1 \\
\hline \multicolumn{3}{|c|}{ Odonata (dragonflies, damselflies) } \\
\hline Enallagma sp. & 3 & 1 \\
\hline Gomphus pallidus & 10 & 1 \\
\hline Pachydiplax longipennis & 8 & 1 \\
\hline
\end{tabular}


Table 11. Benthic invertebrate dip-net survey at transects A, B, and C, Big Four mine-Continued $[-$, no data $]$

\begin{tabular}{ccr}
\hline Species & $\begin{array}{c}\text { January 1983 } \\
\text { count }\end{array}$ & $\begin{array}{r}\text { June } 1983 \\
\text { count }\end{array}$ \\
\hline $\begin{array}{c}\text { Transect A-Continued } \\
\text { Hemiptera (true bugs) } \\
\quad \text { Lethocerus } \\
\text { Diptera (true flies) } \\
\quad \text { Unidentifiable fly larva } \\
\text { Tanypus carinatus }\end{array}$ & 3 & \\
$\begin{array}{c}\text { Coleoptera (beetles) } \\
\text { Bidessus sp. } \\
\text { Hydropopus } \\
\text { Total count }\end{array}$ & 1 & 1 \\
\hline
\end{tabular}

Transect B

Arthropoda

Crustacae

Amphipoda (scuds)

Hyalella azteca

Decopoda (crayfish)

Procambarus sp.

Juvenile crayfish

Insecta

Odonata (dragonflies, damselflies)

Gomphus pallious

Gomphaeschna sp.

$\begin{array}{rr}- & 1 \\ 2 & 1 \\ 18 & 10\end{array}$

Hemiptera (true bugs)

Lethocerus

Coleoptera (beetles)

Bidessus sp.

Hygrotus

14

4

$10 \quad 2$

Gasca

Gastropoda (snails)

Ferrissia sp.

Total count

$4 \quad 2$

43

122

$\frac{1}{54} \quad \overline{\overline{25}}$

Transect C

Arthropoda

Crustacea

Decopoda (shrimp, crayfish)

Palaemonites palludosus

25

12

Juvenile crayfish

18

10

Mullusca

Gastropoda (snails)

Ferrissia sp.

Total count

$\frac{1}{44} \quad \frac{3}{25}$ 


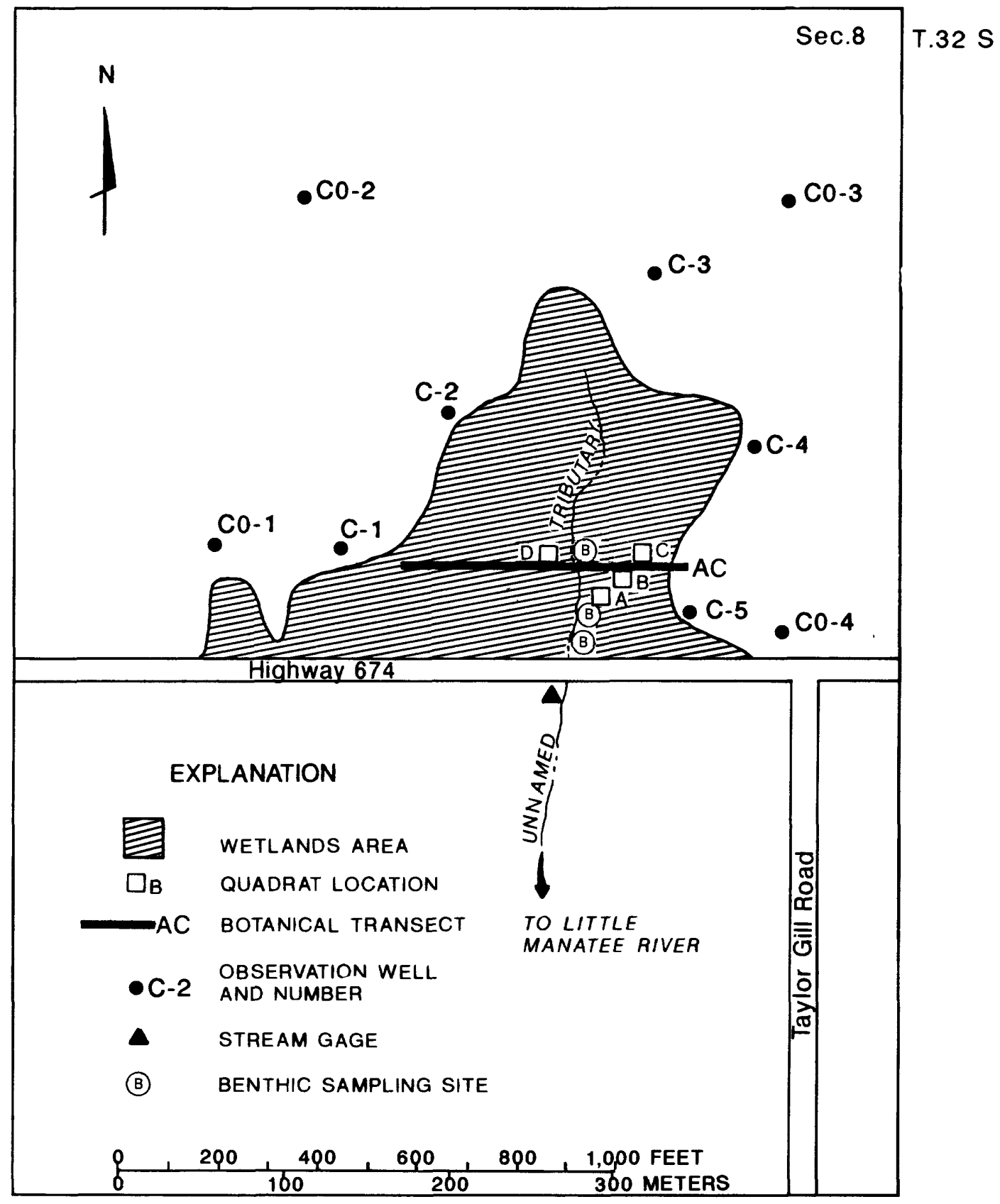

Figure 8. Data-collection network at the control site at the Lonesome mine.

Biology

A transect with biological sampling quadrats, 1 meter square (fig. 8), was established at the control site in April 1984. The quadrats were resurveyed in June 1984, January and July 1985, and January, July, and October 1986. Flora data are given in table 15 . As part of a reconnaissance to evaluate different sampling techniques for the study, three Hester-Dendy ${ }^{1}$ multiplated bottom substrate samplers for benthic invertebrates were installed in the stream in April 1984. Benthic invertebrate samplers were collected after the plates had been in place 1 month. Results of the benthic invertebrate counts are given in table 16 . Because of the intermittent nature of streamflow (table 11), further sampling for benthic invertebrates was discontinued at the control site.

\footnotetext{
${ }^{1}$ Use of brands, firm, or trade names in this report is for identification purposes only and does not constitute endorsement by the U.S. Geological Survey
} 
Table 12. Daily discharge at the unnamed control basin tributary, Lonesome mine, July 1983 through September 1986

[Values are in cubic feet per second; -, no data]

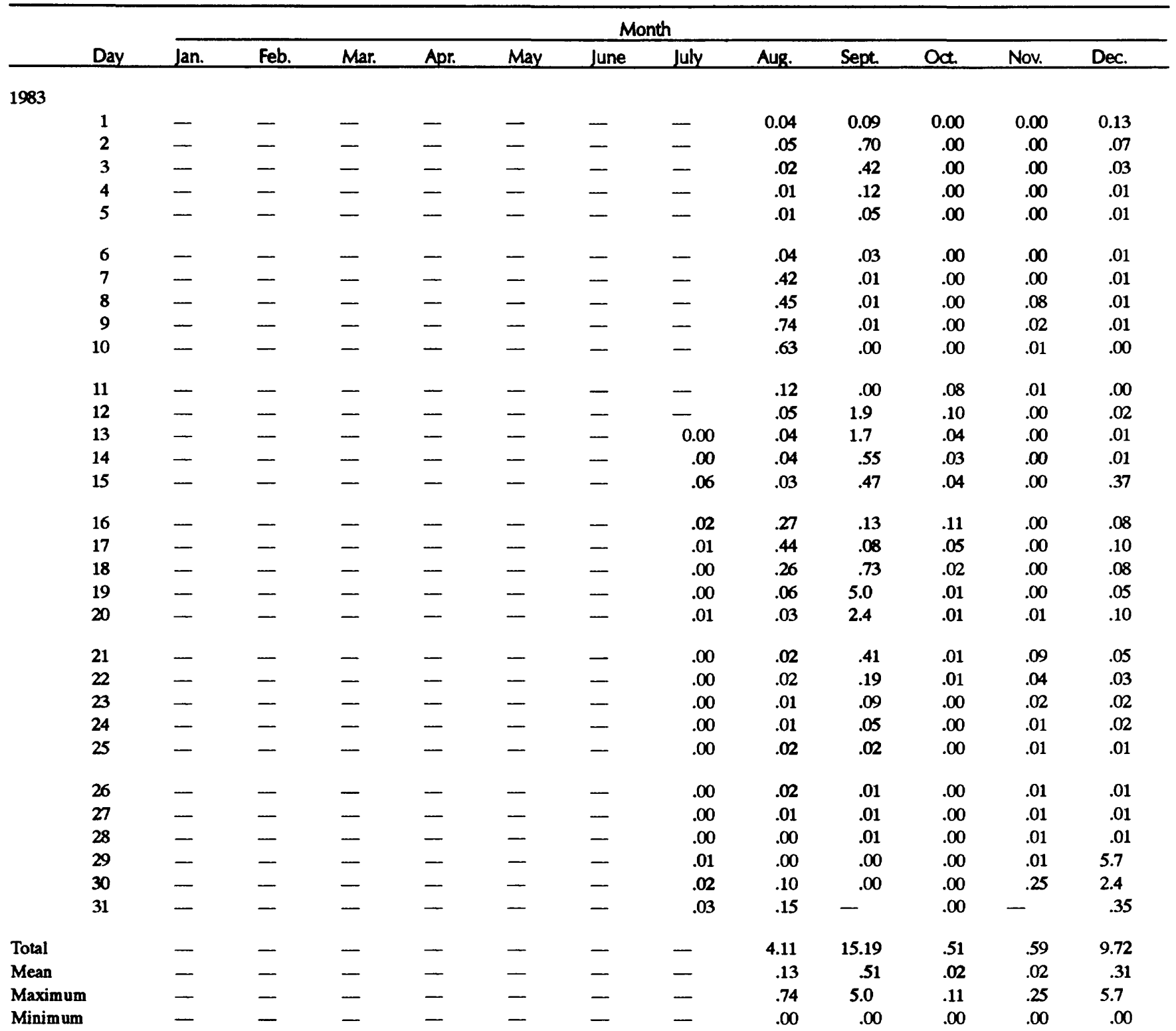

1984

\begin{tabular}{|c|c|c|c|c|c|c|c|c|c|c|c|c|}
\hline 1 & 0.12 & 0.01 & 0.03 & 0.00 & 0.00 & 0.00 & .00 & .48 & .00 & .00 & .00 & .00 \\
\hline 2 & .07 & .01 & .02 & .00 & .00 & .00 & .17 & .42 & .00 & .00 & .00 & .00 \\
\hline 3 & .04 & .01 & .02 & .00 & .00 & .00 & .08 & .12 & .00 & .00 & .00 & .00 \\
\hline 4 & .02 & .01 & .01 & .01 & .00 & .00 & .01 & .13 & .00 & .00 & .00 & .00 \\
\hline 5 & .02 & .01 & .01 & .01 & .00 & .00 & .01 & .04 & .00 & .00 & .00 & .00 \\
\hline 6 & .02 & .01 & .01 & .00 & .00 & .00 & .01 & .02 & .00 & .00 & .00 & .00 \\
\hline 7 & .02 & .00 & .01 & .00 & .00 & .00 & .00 & .01 & .00 & .00 & .00 & .00 \\
\hline 8 & .01 & .00 & .01 & .00 & .00 & .00 & .24 & .01 & .00 & .00 & .00 & .00 \\
\hline 9 & .01 & .00 & .00 & .00 & .00 & .00 & .19 & .00 & .00 & .00 & .00 & .00 \\
\hline 10 & .06 & .00 & .00 & .01 & .00 & .00 & .01 & .00 & .00 & .00 & .00 & .00 \\
\hline 11 & .19 & .00 & .00 & .00 & .00 & .00 & .00 & .00 & .00 & .00 & .00 & .00 \\
\hline 12 & .07 & .00 & .02 & .00 & .00 & .00 & .00 & .00 & .00 & .00 & .00 & .00 \\
\hline 13 & .04 & .48 & .55 & .00 & .00 & .00 & .00 & .00 & .00 & .00 & .00 & .00 \\
\hline 14 & .02 & .27 & .07 & .02 & .00 & .00 & .00 & .01 & .00 & .00 & .00 & .00 \\
\hline 15 & .02 & .08 & .03 & .07 & .00 & .00 & .00 & .00 & .00 & .00 & .00 & .00 \\
\hline
\end{tabular}


Table 12. Daily discharge at the unnamed control basin tributary, Lonesome mine, July 1983 through September 1986-Continued

\begin{tabular}{|c|c|c|c|c|c|c|c|c|c|c|c|c|}
\hline \multirow[b]{2}{*}{ Day } & \multicolumn{12}{|c|}{ Month } \\
\hline & Jan. & Feb. & Mar. & Apr. & May & June & July & Aug. & Sept. & Oct & Nov. & Dec. \\
\hline \multicolumn{13}{|c|}{ 1984-Continued } \\
\hline 16 & 0.02 & 0.04 & 0.01 & 0.03 & 0.00 & 0.00 & 0.01 & 0.00 & 0.00 & 0.00 & 0.00 & 0.00 \\
\hline 17 & .02 & .02 & .01 & .01 & .00 & .69 & .03 & .00 & .00 & .00 & .00 & .00 \\
\hline 18 & .02 & .02 & .01 & .01 & .00 & .04 & .03 & .00 & .00 & .00 & .00 & .00 \\
\hline 19 & .11 & .01 & .00 & .01 & .00 & .01 & .11 & .00 & .00 & .00 & .00 & .00 \\
\hline 20 & .07 & .01 & .00 & .00 & .00 & .00 & .20 & .00 & .00 & .00 & .00 & .00 \\
\hline 21 & .06 & .40 & .00 & .00 & .00 & .00 & .01 & .25 & .00 & .00 & .00 & .00 \\
\hline 22 & .03 & 1.4 & .00 & .00 & .00 & .00 & .01 & .07 & .00 & .00 & .00 & .00 \\
\hline 23 & .03 & .26 & .23 & .00 & .00 & .00 & .01 & .01 & .00 & .00 & .00 & .00 \\
\hline 24 & .02 & .09 & .10 & .00 & .00 & .00 & .07 & .01 & .00 & .00 & .00 & .00 \\
\hline 25 & .02 & .05 & .04 & .00 & .00 & .00 & .12 & .01 & .00 & .00 & .00 & .00 \\
\hline 26 & .02 & .03 & .02 & .00 & .00 & .00 & .50 & .02 & .00 & .00 & .00 & .00 \\
\hline 27 & .02 & .08 & .01 & .00 & .00 & .00 & .35 & .01 & .01 & .00 & .00 & .00 \\
\hline 28 & .02 & .22 & .01 & .00 & .00 & .00 & .06 & .00 & .04 & .00 & .00 & .00 \\
\hline 29 & .01 & .06 & .01 & .00 & .00 & .00 & .02 & .00 & .00 & .00 & .00 & .00 \\
\hline 30 & .01 & - & .01 & .00 & .00 & .00 & .59 & .00 & .00 & .00 & .00 & .00 \\
\hline 31 & .01 & - & .01 & - & .00 & - & .37 & .00 & - & .00 & - & .00 \\
\hline Total & 1.22 & 3.58 & 1.26 & .18 & .00 & .74 & 3.21 & 1.62 & .05 & .00 & .00 & .00 \\
\hline Mean & .04 & .12 & .04 & .01 & .00 & .02 & .10 & .05 & .00 & .00 & .00 & .00 \\
\hline Maximum & .19 & 1.4 & .55 & .07 & .00 & .69 & .59 & .48 & .04 & .00 & .00 & .00 \\
\hline Minimum & .01 & .00 & .00 & .00 & .00 & .00 & .00 & .00 & .00 & .00 & .00 & .00 \\
\hline \multicolumn{13}{|l|}{1985} \\
\hline 1 & .00 & .00 & .00 & .00 & .00 & .00 & .00 & .00 & 6.2 & .01 & .12 & .00 \\
\hline 2 & .00 & .00 & .00 & .00 & .00 & .00 & .00 & .00 & .46 & .00 & .02 & .00 \\
\hline 3 & .00 & .00 & .00 & .00 & .00 & .00 & .00 & .00 & .08 & .33 & .01 & .00 \\
\hline 4 & .00 & .00 & .00 & .00 & .00 & .00 & .00 & .00 & .13 & .14 & .01 & .00 \\
\hline 5 & .00 & .00 & .00 & .00 & .00 & .00 & .00 & .00 & .02 & .58 & .01 & .00 \\
\hline 6 & .00 & .00 & .00 & .00 & .00 & .00 & .00 & .00 & .02 & .81 & .00 & .01 \\
\hline 7 & .00 & .00 & .00 & .00 & .00 & .00 & .00 & .09 & .01 & .03 & .00 & .00 \\
\hline 8 & .00 & .00 & .00 & .00 & .00 & .00 & .00 & .04 & .01 & .01 & .00 & .00 \\
\hline 9 & .00 & .00 & .00 & .00 & .00 & .00 & .00 & .07 & .01 & .01 & .00 & .00 \\
\hline 10 & .00 & .00 & .00 & .00 & .00 & .00 & .00 & .04 & .00 & .01 & .00 & .00 \\
\hline 11 & .00 & .00 & .00 & .00 & .00 & .00 & .00 & .01 & .00 & .01 & .00 & .00 \\
\hline 12 & .00 & .00 & .00 & .00 & .00 & .00 & .00 & .00 & .00 & .00 & .00 & .00 \\
\hline 13 & .00 & .00 & .00 & .00 & .00 & .00 & .00 & .00 & .00 & .00 & .00 & .00 \\
\hline 14 & .00 & .00 & .00 & .00 & .00 & .01 & .00 & .00 & .00 & .00 & .00 & .01 \\
\hline 15 & .00 & .00 & .00 & .00 & .00 & .03 & .00 & .01 & .00 & .00 & .00 & .00 \\
\hline 16 & .00 & .00 & .00 & .00 & .00 & .01 & .00 & .01 & .00 & .00 & .00 & .00 \\
\hline 17 & .00 & .00 & .00 & .00 & .00 & .00 & .00 & .01 & .00 & .00 & .00 & .00 \\
\hline 18 & .00 & .00 & .00 & .00 & .00 & .00 & .00 & .01 & .00 & .00 & .00 & .00 \\
\hline 19 & .00 & .00 & .00 & .00 & .00 & .00 & .00 & .01 & .00 & .00 & .00 & .00 \\
\hline 20 & .00 & .00 & .00 & .00 & .00 & .00 & .21 & .00 & .01 & .00 & .00 & .00 \\
\hline 21 & .00 & .00 & .02 & .00 & .00 & .00 & .01 & .00 & .01 & .00 & .00 & .00 \\
\hline 22 & .00 & .00 & .01 & .00 & .00 & .00 & .00 & .00 & .01 & .00 & .00 & .00 \\
\hline 23 & .00 & .00 & .00 & .00 & .00 & .00 & .00 & .00 & .00 & .00 & .00 & .00 \\
\hline 24 & .00 & .00 & .00 & .00 & .00 & .00 & .00 & .00 & .00 & .00 & .00 & .00 \\
\hline 25 & .00 & .00 & .00 & .00 & .00 & .00 & .00 & .01 & .00 & .00 & .00 & .01 \\
\hline 26 & .00 & .00 & .00 & .00 & .00 & .00 & .00 & .00 & .01 & .00 & .00 & .00 \\
\hline 27 & .00 & .00 & .00 & .00 & .00 & .00 & .00 & .00 & .01 & .00 & .00 & .00 \\
\hline 28 & .00 & .00 & .00 & .00 & .00 & .00 & .00 & .01 & .01 & .00 & .00 & .00 \\
\hline 29 & .00 & - & .00 & .00 & .00 & .00 & .00 & .53 & .01 & .00 & .00 & .00 \\
\hline 30 & .00 & - & .00 & .00 & .00 & .00 & .00 & .12 & .01 & .00 & .00 & .00 \\
\hline 31 & .00 & - & .00 & - & .00 & - & .00 & 1.1 & - & .01 & - & .00 \\
\hline
\end{tabular}


Table 12. Daily discharge at the unnamed control basin tributary, Lonesome mine, July 1983 through September 1986-Continued

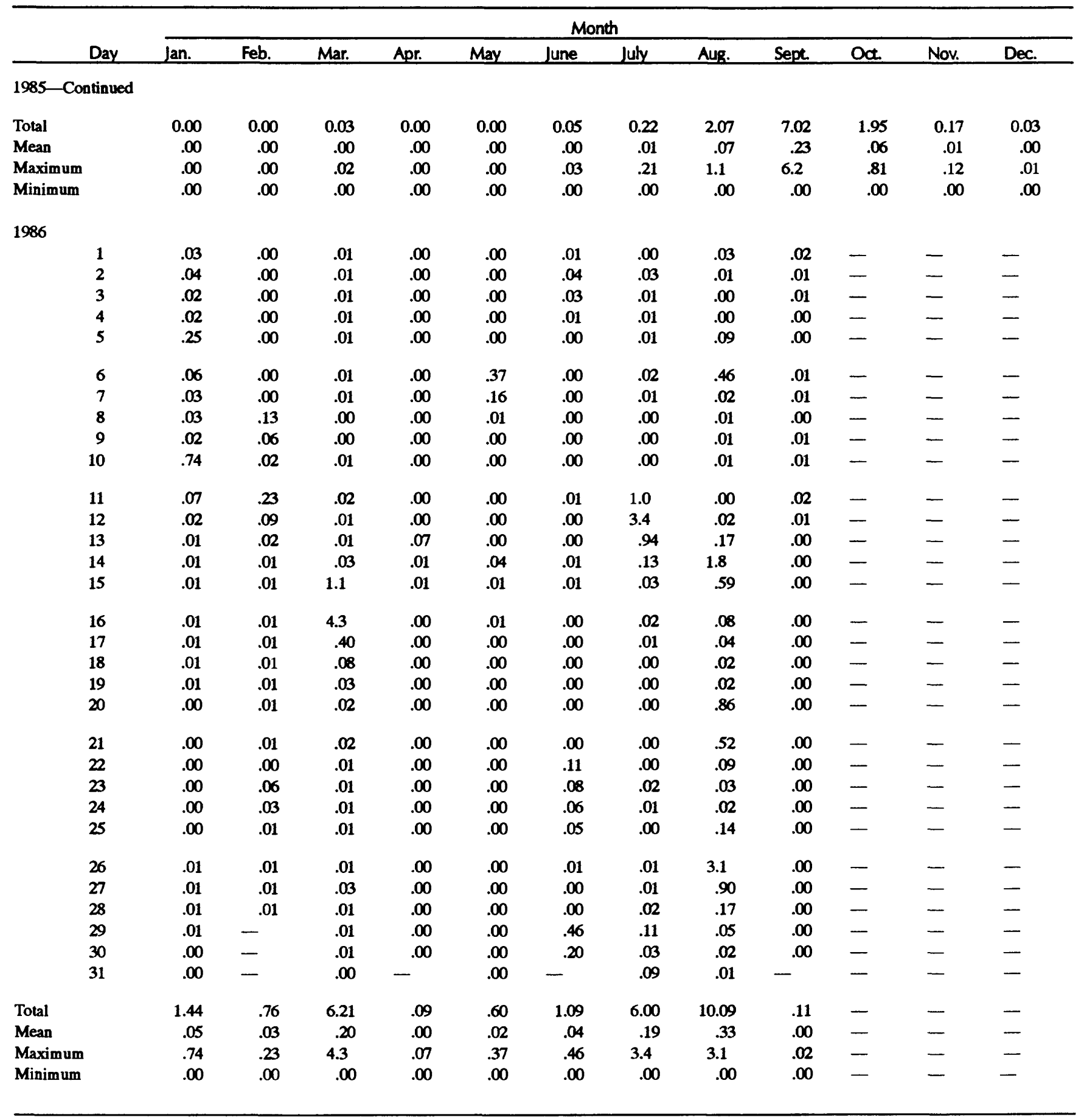



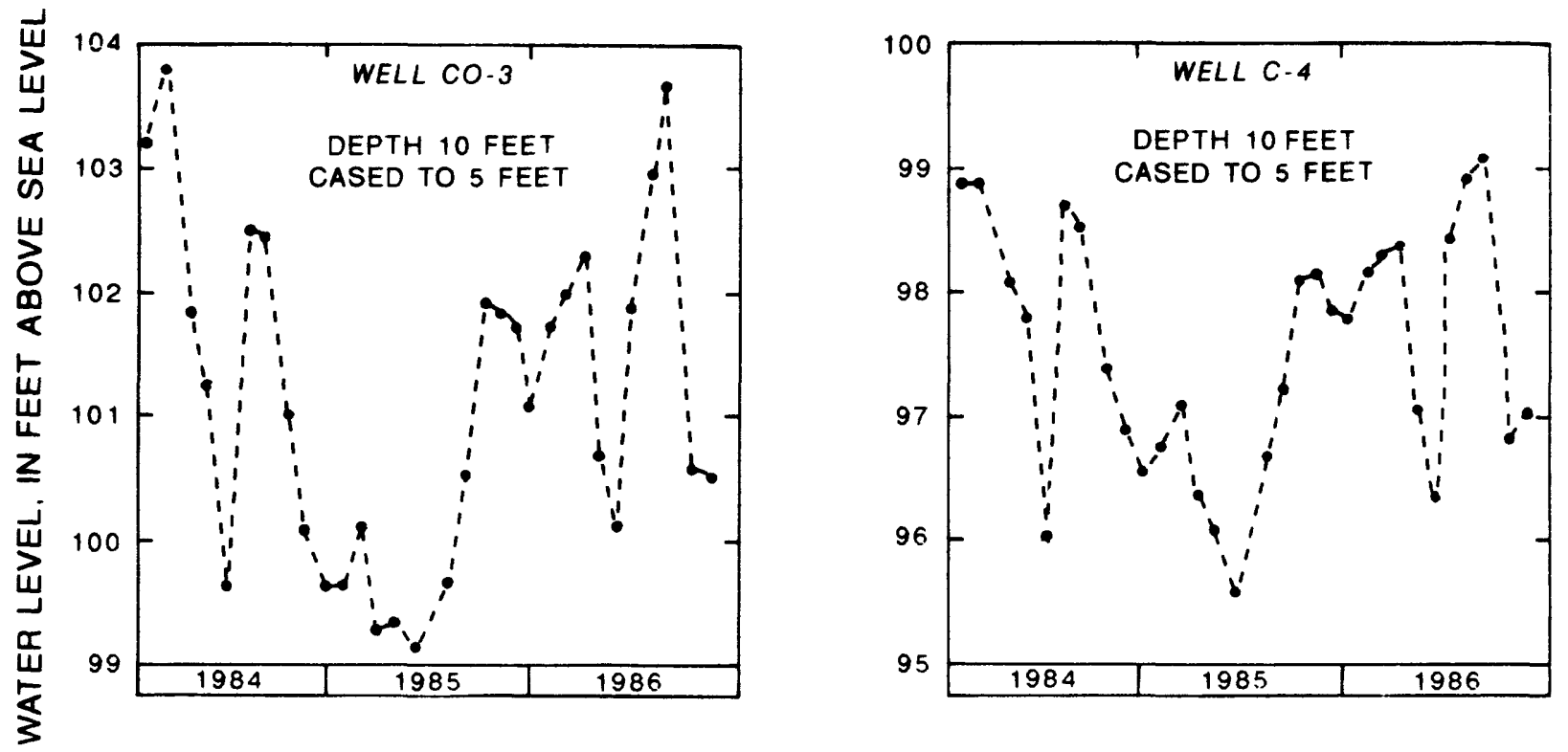

Figure 9. Water levels at wells $\mathrm{CO}-3$ and $\mathrm{C}-4$ at the control basin, Lonesome mine.

Table 13. Water-level observations in the surficial aquifer system at the Lonesome mine near Fort Lonesome

[Water-level altitudes are in feet above sea level; --, no data]

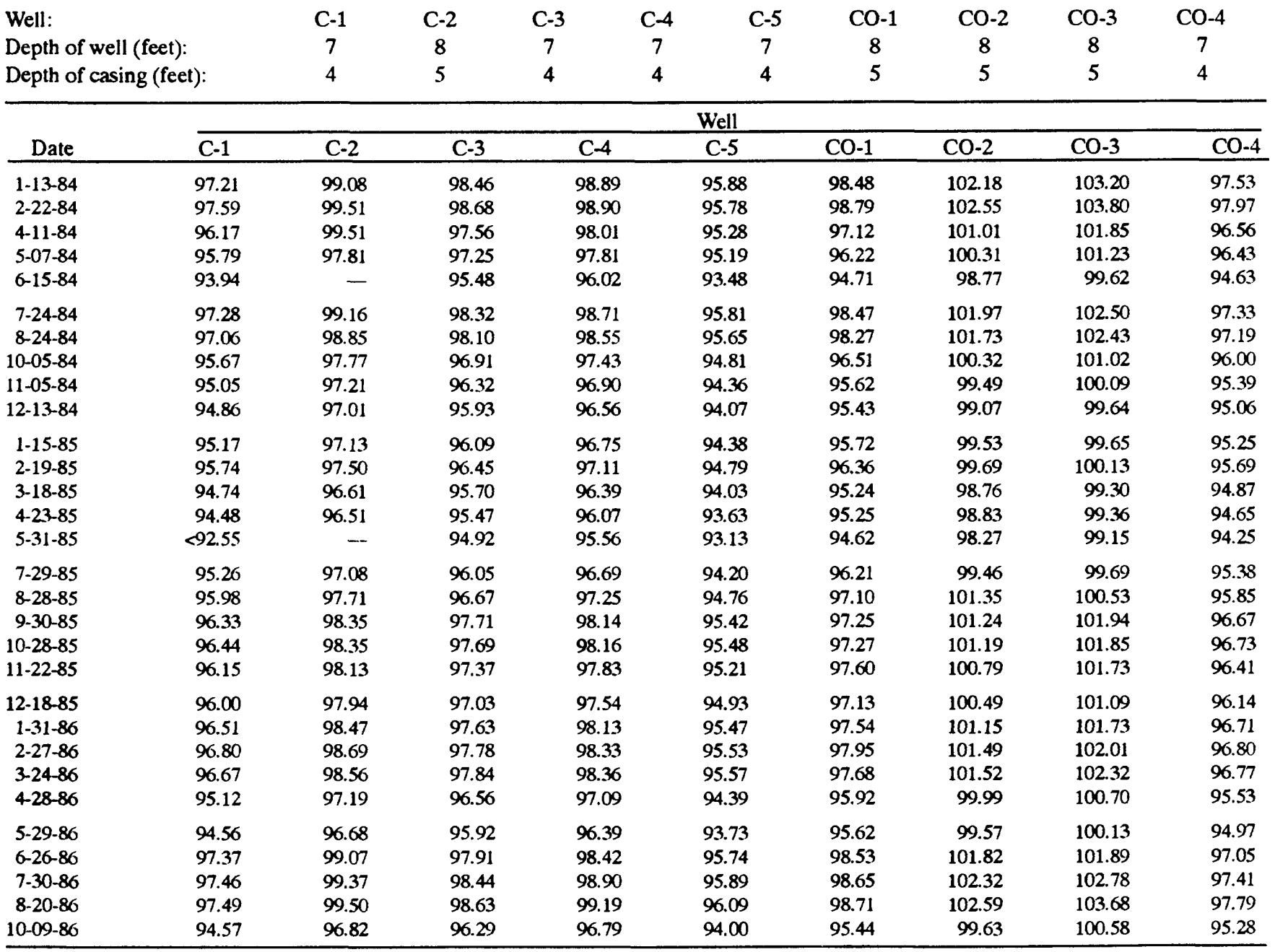


Table 14. Water-quality data from the unnamed tributary, Lonesome mine, September 23, 1983

[Station number, 274215082072000 . $\mathrm{ft}^{3} / \mathrm{s}$, cubic feet per second; $\mu \mathrm{S} / \mathrm{cm}$, microsiemens per centimeter, $\mathrm{mg} /$, milligrams per liter, $\mu \mathrm{g} /$, micrograms per liter, $\mathrm{PCi} / \mathrm{L}$, picocuries per liter]

\begin{tabular}{|c|c|c|c|}
\hline $\begin{array}{l}\text { Constituents and } \\
\text { physical properties }\end{array}$ & Value & $\begin{array}{l}\text { Constituents and } \\
\text { physical properties }\end{array}$ & Value \\
\hline Streamflow, instantaneous $\left(\mathrm{ft}^{3} / \mathrm{s}\right)$ & 0.07 & Lead, dissolved ( $\mu g / L$ as $P b)$ & 2 \\
\hline Specific conductance $(\mu \mathrm{S} / \mathrm{cm})$ & 86 & Manganese, dissolved ( $\mu g / \mathrm{L}$ as $\mathrm{Mn})$ & 8 \\
\hline pH (standard units) & 5.30 & Molybdenum, dissolved ( $\mu \mathrm{g} / \mathrm{L}$ as $\mathrm{Mo}$ ) & $<1$ \\
\hline $\mathrm{pH}$, lab (standard units) & 5.70 & Nickel, dissolved ( $\mu g / L$ as $\mathrm{Ni})$ & $<1$ \\
\hline Nitrogen, ammonia, dissolved (mg/L as $\mathrm{N}$ ) & 0.030 & Vanadium, dissolved ( $\mu \mathrm{g} / \mathrm{L}$ as $\mathrm{V})$ & 7 \\
\hline Nitrogen, ammonia plus organic, total (mg/L as $\mathrm{N}$ ) & 1.7 & Zinc, dissolved ( $\mu \mathrm{g} / \mathrm{L}$ as $\mathrm{Zn})$ & 8 \\
\hline Nitrogen, $\mathrm{NO}_{2}+\mathrm{NO}_{3}$, dissolved (mg/L as $\mathrm{N}$ ) & 0.120 & Aluminum, dissolved ( $\mu \mathrm{g} / \mathrm{L}$ as $\mathrm{Al})$ & 410 \\
\hline Phosphate, ortho, dissolved $\left(\mathrm{mg} / \mathrm{L}\right.$ as $\left.\mathrm{PO}_{4}\right)$ & 2.1 & Gross beta, dissolved ( $\mathrm{pC} \mathrm{i} / \mathrm{L}$ as $\mathrm{CS}-137$ ) & 5.6 \\
\hline Phosphorus, total (mg/L as P) & 0.780 & Radium 226, dissolved, radon method ( $\mathrm{pCi} / \mathrm{L})$ & 0.48 \\
\hline Phosphorus, dissolved (mg/Las $\mathrm{P}$ ) & 0.680 & Iodide, dissolved (mg/L as I) & 0.002 \\
\hline Phosphorus, ortho, dissolved (mg/L as P) & 0.690 & Bromide, dissolved (mg/L as $\mathrm{Br}$ ) & 0.11 \\
\hline Boron, dissolved ( $\mu \mathrm{g} / \mathrm{L}$ as $\mathrm{B})$ & 160 & Mercury, dissolved ( $\mu \mathrm{g} / \mathrm{L}$ as $\mathrm{Hg})$ & 0.3 \\
\hline Cadmium, dissolved ( $\mu \mathrm{g} / \mathrm{L}$ as $\mathrm{Cd}$ ) & $<1$ & Uranium, dissolved, extraction ( $\mu g / L)$ & 0.15 \\
\hline Chromium, dissolved ( $\mu \mathrm{g} / \mathrm{L}$ as $\mathrm{Cr}$ ) & 20 & Gross alpha, dissolved ( $\mu g / L$ as U-nat) & 5.5 \\
\hline Copper, dissolved ( $\mu \mathrm{g} / \mathrm{L}$ as $\mathrm{Cu}$ ) & $<1$ & Gross beta, dissolved ( $\mathrm{pCi} / \mathrm{L}$ as $\mathrm{Sr} / \mathrm{YT}-90$ ) & 4.8 \\
\hline
\end{tabular}

Table 15. Composition of flora at transect AC, Lonesome mine, 1984 through 1986

$[-$, no data $]$

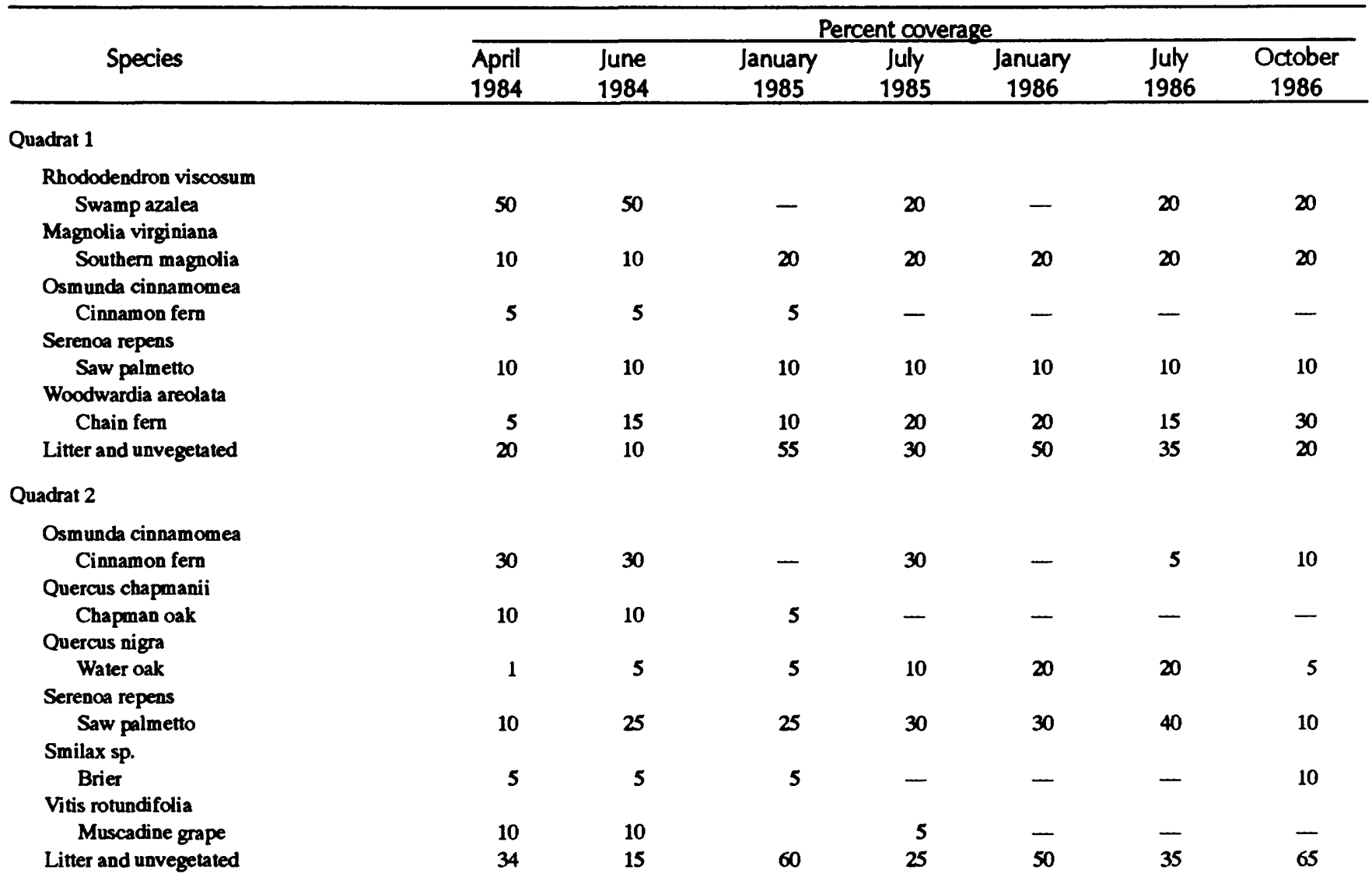


Table 15. Composition of flora at transect $A C$, Lonesome mine, 1984 through 1986—Continued $[-$, no data $]$

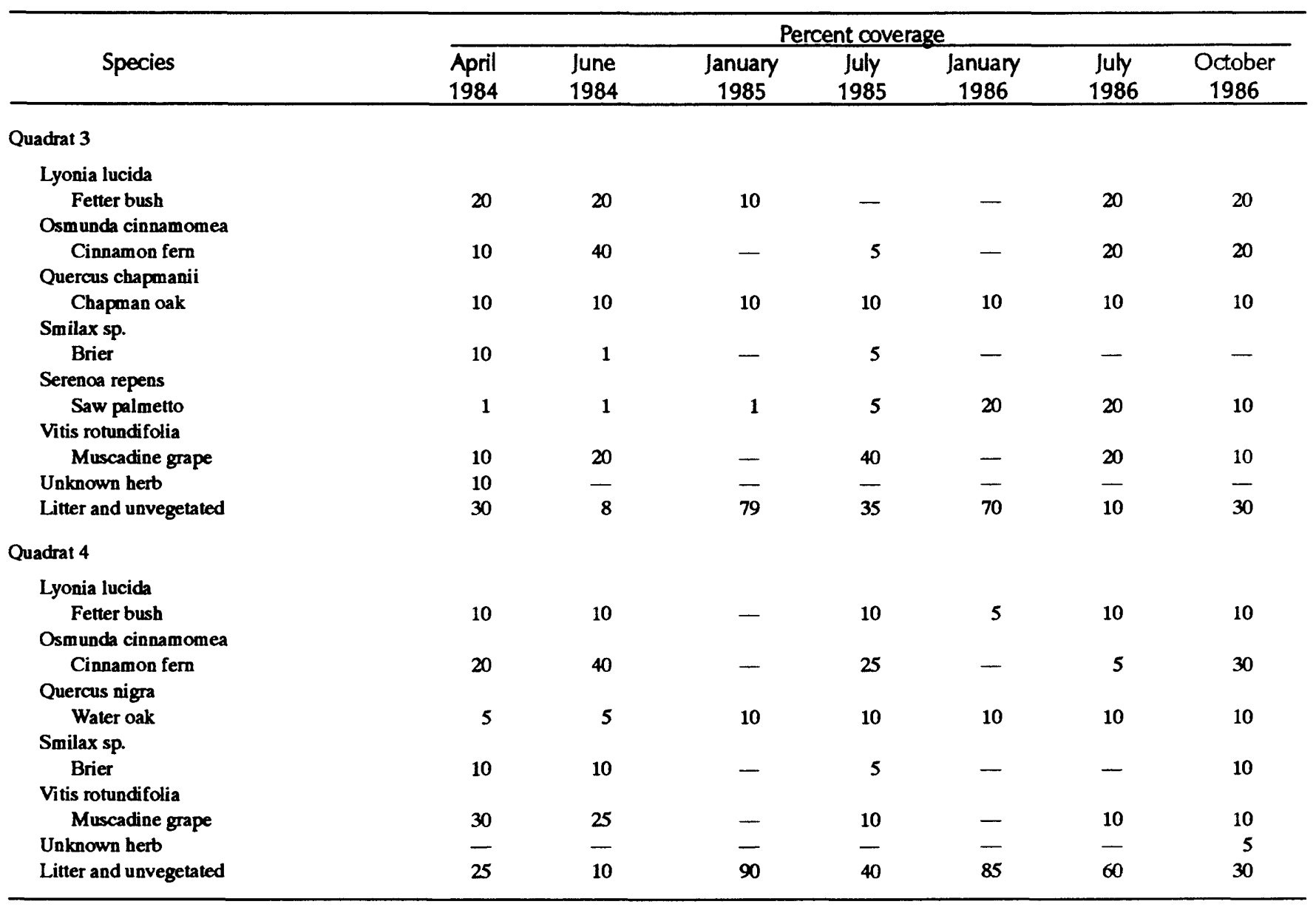

Table 16. Results of benthic invertebrate sampling using Hester-Dendy multiplated samplers at the unnamed tributary, Lonesome mine, April 1984

Arthropoda

Crustacea

\begin{tabular}{|c|c|}
\hline Amphipoda (scuds) & \\
\hline Hyalella azteca & 1 \\
\hline Isopoda (sow bugs) & \\
\hline Unidentifiable isopod & 1 \\
\hline Insecta & \\
\hline Odonata (dragonflies, dams & \\
\hline Enallagma sp. & 1 \\
\hline Gomphus pallidus & 1 \\
\hline Diptera (true flies) & \\
\hline Chronomidae & \\
\hline Tanypus carinatus & 25 \\
\hline Unidentifiable fly larva & $\underline{5}$ \\
\hline Total count & 34 \\
\hline
\end{tabular}




\section{SUMMARY}

Hydrologic and biologic data were collected from two small basins in southeast Hillsborough County. The test basin at the Big Four Mine was monitored starting in July 1982 until mining began in November 1983 . Following regrading of the 14 acres of wetlands area that was mined, a groundwater monitoring network was reestablished at the site in July 1985 and operated through September 1986. Data collection at the control site basin at the Lonesome mine began in September 1983 and continued through September 1986. Streamflow, rainfall, and ground-water level data for the period of record, through September 1986, are presented along with lithologic descriptions from two core holes and soil sample analysis from three cross sections at the test basin. Surface-water and ground-water quality data and biologic data also are presented.

\section{SELECTED REFERENCES}

Biological Research Associates, 1982, Ecological evaluation of proposed mine land for AMAX Phosphates Inc., Big Four Mine, Bradley, Florida: Consultant's report on file at U.S. Geological Survey, Tampa, Fla., 136 p.
Corral, M.A., Jr., and Wolansky, R.M., 1984, Generalized thickness and configuration of the top of the intermediate aquifer, west-central Florida: U.S. Geological Survey Water-Resources Investigations Report 84-4018, 1 sheet.

Jackson, M.L., 1975, Soil chemical analysis advance course (2d ed.): Madison, University of Wisconsin, 895 p.

Miller, J.A., 1986, Hydrogeologic framework of the Floridan aquifer system in Florida and in parts of Georgia, Alabama, and South Carolina: U.S. Geological Survey Professional Paper 1403-B, 91 p.

Ryder, P.D., 1982, Digital model of predevelopment flow in the Tertiary limestone (Floridan) aquifer system in west-central Florida: U.S. Geological Survey Water-Resources Investigations 81-54, 61 p.

___ 1985, Hydrology of the Floridan aquifer system in west-central Florida: U.S. Geological Survey Professional Paper 1403-F, 63 p.

Southeastern Geological Society, 1986, Hydrogeological units of Florida: Florida Geological Survey Special Publication 28, 9 p.

Stowasser, W.F., 1986, Phosphate rock, in Metals and Minerals: U.S. Bureau of Mines 1986 Minerals Yearbook, v. 1, p. 723-731.

White, W.A., 1970, The geomorphology of the Florida peninsula: Florida Bureau of Geology Bulletin 51, 164 p.

Wolansky, R.M., 1983, Hydrogeology of the Sarasota-Port Charlotte area, Florida: U.S. Geological Survey Water-Resources Investigations Report 82-4089, 48 p. 